\title{
Asymptotic Analysis of the Eigenvalues of a Laplacian Problem in a Thin Multidomain
}

\author{
Antonio Gaudiello*and Ali Sili ${ }^{\dagger}$
}

\begin{abstract}
We consider a thin multidomain of $\mathbb{R}^{N}, N \geq 2$, consisting of two vertical cylinders, one placed upon the other: the first one with given height and small cross section, the second one with small thickness and given cross section. In this multidomain we study the asymptotic behavior, when the volumes of the two cylinders vanish, of a Laplacian eigenvalue problem and of a $L^{2}$-Hilbert orthonormal basis of eigenvectors. We derive the limit eigenvalue problem (which is well posed in the union of the limit domains, with respective dimension 1 and $N-1$ ) and the limit basis. We discuss the limit models and we precise how these limits depend on the dimension $N$ and on limit $q$ of the ratio between the volumes of the two cylinders.
\end{abstract}

Keywords: Laplacian eigenvalue problem; thin multidomains; dimension reduction. 2000 AMS subject classifications: 49R50, 35P20, 74K05, 74K30, 74K35.

\section{Introduction and main results}

For every $n \in \mathbb{N}$, let $\Omega_{n} \subset \mathbb{R}^{N}, N \geq 2$, be a thin multidomain consisting of two vertical cylinders, one placed upon the other: the first one with constant height 1 and small cross section $r_{n} \omega$, the second one with small thickness $h_{n}$ and constant cross section $\omega$, where $\omega$ is a bounded open connected set of $\mathbb{R}^{N-1}$ containing the origin $0^{\prime}$ of $\mathbb{R}^{N-1}$ and with smooth boundary, $r_{n}$ and $h_{n}$ are two small parameters converging to zero. Precisely,

$$
\Omega_{n}=\left(r_{n} \omega \times\left[0,1[) \bigcup(\omega \times]-h_{n}, 0[)\right.\right.
$$

(for instance, see Fig. 1 for $N=2$ and Fig. 2 for $N=3$ ).

In $\Omega_{n}$ consider the following eigenvalue problem:

$$
\left\{\begin{array}{l}
-\Delta U_{n}=\lambda U_{n} \text { in } \Omega_{n}, \\
U_{n}=0 \text { on } \Gamma_{n}=\left(r_{n} \omega \times\{1\}\right) \cup(\partial \omega \times]-h_{n}, 0[), \\
\frac{\partial U_{n}}{\partial \nu}=0 \text { on } \partial \Omega_{n} \backslash \Gamma_{n},
\end{array}\right.
$$

*DAEIMI, Università degli Studi di Cassino, via G. Di Biasio 43, 03043 Cassino (FR), Italia. e-mail: gaudiell@unina.it

†Département de Mathématiques, Université du Sud Toulon-Var, BP 20132, 83957 La Garde cedex, France, \& LATP, UMR 6632, Université de Provence, 39 rue F. Joliot-Curie, 13453 Marseille cedex 13, France. e-mail: sili@univ-tln.fr 


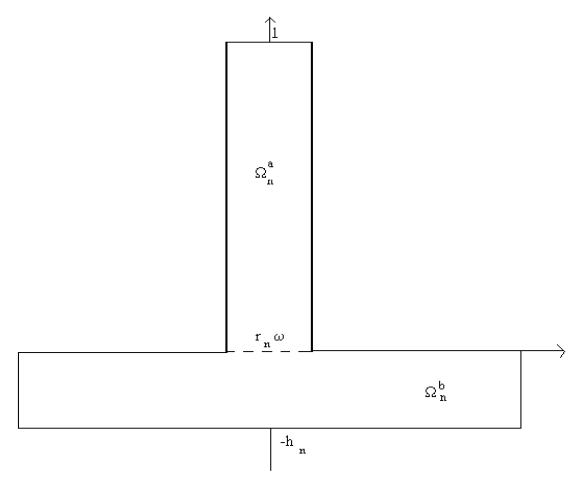

Figure 1: the thin multidomain for $N=2$.

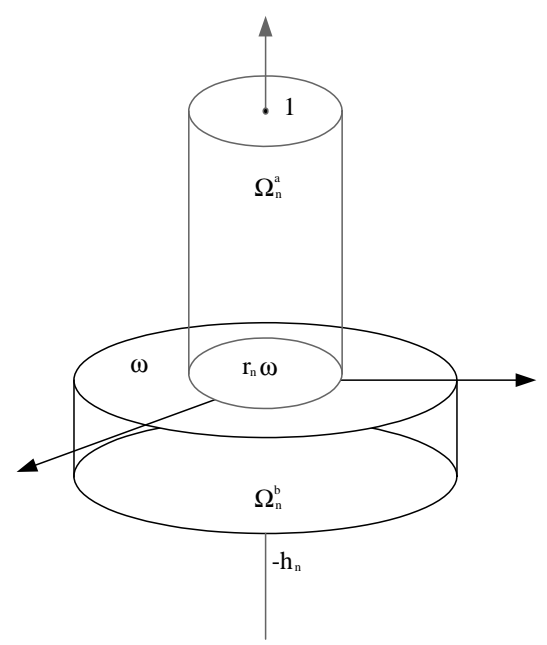

Figure 2: the thin multidomain for $N=3$.

where $\nu$ denotes the exterior unit normal to $\Omega_{n}$. Remark that $r_{n} \omega \times\{1\}$ is the top of the upper cylinder, while $\partial \omega \times]-h_{n}, 0[$ is the lateral surface of the second one.

It is well known (for instance, see Th. 6.2-1 in [15]) that, for every $n \in \mathbb{N}$, there exists an increasing diverging sequence of positive numbers $\left\{\lambda_{n, k}\right\}_{k \in \mathbb{N}}$ and a $L^{2}\left(\Omega_{n}\right)$-Hilbert orthonormal basis $\left\{U_{n, k}\right\}_{k \in \mathbb{N}}$, such that $\left\{\lambda_{n, k}\right\}_{k \in \mathbb{N}}$ forms the set of all the eigenvalues of Problem (1.11) and, for every $k \in \mathbb{N}, U_{n, k} \in \mathcal{V}_{n}=\left\{V \in H^{1}\left(\Omega_{n}\right): V=0\right.$ on $\left.\Gamma_{n}\right\}$ is an eigenvector of (1.1) with eigenvalue $\lambda_{n, k}$. Moreover, $\left\{\lambda_{n, k}^{-\frac{1}{2}} U_{n, k}\right\}_{k \in \mathbb{N}}$ is a $\mathcal{V}_{n}$-Hilbert orthonormal basis, by equipping $\mathcal{V}_{n}$ with the inner product: $(U, V) \in \mathcal{V}_{n} \times \mathcal{V}_{n} \rightarrow \int_{\Omega_{n}} D U D V d x$.

The aim of this paper is to study the asymptotic behavior of the sequences $\left\{\left(\lambda_{n, k}, U_{n, k}\right)\right\}_{n \in \mathbb{N}}$, for every $k \in \mathbb{N}$, as $n$ diverges.

We derive the limit eigenvalue problem (which is well posed in the union of the limit 
domains: ]0, 1[ and $\omega$, with respective dimension: 1 and $N-1$ ) and the limit basis, and we precise how these limits depend on the dimension $N$ and on limit $q=\lim _{n} \frac{h_{n}}{r_{n}^{N-1}}$ of the ratio between the volumes of the two cylinders.

In the sequel, $D$ stands for the gradient. Moreover, $x=\left(x_{1}, \cdots, x_{N-1}, x_{N}\right)=\left(x^{\prime}, x_{N}\right)$ denotes the generic point of $\mathbb{R}^{N}$, and $D_{x^{\prime}}$ and $D_{x_{N}}$ stand for the gradient with respect to the first $N-1$ variables and for the derivative with respect to the last variable, respectively. Furthermore, if $u$ depends only on one variable, $u^{\prime}$ and $u^{\prime \prime}$ stand for the first and second derivative, respectively.

If $h_{n} \simeq r_{n}^{N-1}$, we obtain the following main result:

Theorem 1.1. Assume that

$$
\left.\lim _{n} \frac{h_{n}}{r_{n}^{N-1}}=q \in\right] 0,+\infty[.
$$

Then, there exists an increasing diverging sequence of positive numbers $\left\{\lambda_{k}\right\}_{k \in \mathbb{N}}$ such that

$$
\lim _{n} \lambda_{n, k}=\lambda_{k}, \quad \forall k \in \mathbb{N},
$$

and $\left\{\lambda_{k}\right\}_{k \in \mathbb{N}}$ is the set of all the eigenvalues of the following problem:

$$
\left\{\begin{array}{l}
\left.-u^{a \prime \prime}=\lambda u^{a} \text { in }\right] 0,1[, \\
-\Delta u^{b}=\lambda u^{b} \text { in } \omega, \\
u^{a}(1)=0, \quad u^{a \prime}(0)=0, \\
u^{b}=0 \text { on } \partial \omega,
\end{array}\right.
$$

if $N \geq 3$; of the following one:

$$
\left\{\begin{array}{l}
\left.-u^{a \prime \prime}=\lambda u^{a} \text { in }\right] 0,1[ \\
\left.-u^{b^{\prime \prime}}=\lambda u^{b} \text { in }\right] c, 0[ \\
\left.-u^{b^{\prime \prime}}=\lambda u^{b} \text { in }\right] 0, d[ \\
u^{a}(1)=0, \\
u^{b}(c)=0, \quad u^{b}(d)=0 \\
u^{a}(0)=u^{b}(0) \\
|\omega| u^{a \prime}(0)=q\left(u^{b^{\prime}}\left(0^{-}\right)-u^{b^{\prime}}\left(0^{+}\right)\right)
\end{array}\right.
$$

if $N=2$ and $\omega=] c, d[$. 
There exists an increasing sequence of positive integer numbers $\left\{n_{i}\right\}_{i \in \mathbb{N}}$ and a sequence $\left\{\left(u_{k}^{a}, u_{k}^{b}\right)\right\}_{k \in \mathbb{N}} \subset V$ (depending possibly on the selected subsequence $\left\{n_{i}\right\}_{i \in \mathbb{N}}$ ), where $V=\left\{\left(v^{a}, v^{b}\right) \in H^{1}(] 0,1[) \times H^{1}(\omega): v^{a}(1)=0, v^{b}=0\right.$ on $\partial \omega,\left(\right.$ and $v^{a}(0)=v^{b}(0)$ if $\left.\left.N=2\right)\right\}$, such that

$$
\begin{gathered}
\lim _{i}\left(\int_{\left.r_{n_{i}} \omega \times\right] 0,1[}\left|U_{n_{i}, k}-\frac{u_{k}^{a}}{r_{n_{i}}^{\frac{N-1}{2}}}\right|^{2}+\left|D_{x^{\prime}} U_{n_{i}, k}\right|^{2}+\left|\partial_{x_{N}} U_{n_{i}, k}-\frac{u_{k}^{a \prime}}{r_{n_{i}}^{\frac{N-1}{2}}}\right|^{2} d x\right)=0, \\
\lim _{i}\left(\int_{\omega \times]-h_{n_{i}}, 0[}\left|U_{n_{i}, k}-\frac{q^{\frac{1}{2}}}{h_{n_{i}}^{\frac{1}{2}}} u_{k}^{b}\right|^{2}+\left|D_{x^{\prime}} U_{n_{i}, k}-\frac{q^{\frac{1}{2}}}{h_{n_{i}}^{\frac{1}{2}}} D u_{k}^{b}\right|^{2}+\left|\partial_{x_{N}} U_{n_{i}, k}\right|^{2} d x\right)=0 .
\end{gathered}
$$

as $i \rightarrow+\infty$, for every $k \in \mathbb{N}$, and $u_{k}=\left(u_{k}^{a}, u_{k}^{b}\right)$ is an eigenvector of Problem (1.3) if $N \geq 3$ (Problem 1.4) if $N=2$ ) with eigenvalue $\lambda_{k}$

Moreover, $\left\{u_{k}\right\}_{k \in \mathbb{N}}$ is a $L^{2}(] 0,1[) \times L^{2}(\omega)$-orthonormal basis with respect to the inner product: $|\omega| \int_{0}^{1} u^{a} v^{a} d x_{N}+q \int_{\omega} u^{b} v^{b} d x^{\prime}$, and $\left\{\lambda_{k}^{-\frac{1}{2}} u_{k}\right\}_{k \in \mathbb{N}}$ is a $V$-Hilbert orthonormal basis with respect to the inner product: $|\omega| \int_{0}^{1} u^{a \prime} v^{a \prime} d x_{N}+q \int_{\omega} D u^{b} D v^{b} d x^{\prime}$.

If $N \geq 3$, consider the following problems:

$$
\left\{\begin{array}{l}
\left.-u^{a \prime \prime}=\lambda^{a} u^{a} \text { in }\right] 0,1[, \\
u^{a}(1)=0, \quad u^{a \prime}(0)=0,
\end{array}\right.
$$

and

$$
\left\{\begin{array}{l}
-\Delta u^{b}=\lambda^{b} u^{b} \text { in } \omega, \\
u^{b}=0 \text { on } \partial \omega
\end{array}\right.
$$

By denoting with $\left\{\lambda_{k}^{a}\right\}_{k \in \mathbb{N}}$ the increasing sequence of all the eigenvalues of Problem (1.7) (i.e. $\left\{\left(\frac{\pi}{2}+k \pi\right)^{2}\right\}_{k \in \mathbb{N}_{0}}$, where $\mathbb{N}_{0}=\mathbb{N} \cup\{0\}$ ), and with $\left\{\lambda_{k}^{b}\right\}_{k \in \mathbb{N}}$ the increasing sequence of all the eigenvalues of Problem (1.8), it is easily seen that:

$$
\left\{\lambda_{k}: k \in \mathbb{N}\right\}=\left\{\lambda_{k}^{a}: k \in \mathbb{N}\right\} \cup\left\{\lambda_{k}^{b}: k \in \mathbb{N}\right\} .
$$

As regards as the multiplicity, if $\lambda$ is an eigenvalue only of Problem (1.7), then it is a simple eigenvalue of Problem (1.3) with eigenvector $\left(\cos \left(\frac{\pi}{2}+k \pi\right) x_{N}, 0\right)$, for some $k \in \mathbb{N}_{0}$. If $\lambda$ is an eigenvalue only of Problem (1.8) with multiplicity $s$ (and with linearly independent eigenvectors: $\left.w_{1}^{b}, \cdots, w_{s}^{b}\right)$, then it is an eigenvalue of Problem (1.3) with multiplicity $s$ and with linearly independent eigenvectors: $\left(0, w_{1}^{b}\right), \cdots,\left(0, w_{s}^{b}\right)$. If $\lambda$ is an eigenvalue of both Problem (1.8) with multiplicity $s$ (and with linearly independent eigenvectors: $w_{1}^{b}, \cdots, w_{s}^{b}$ ) 
and Problem (1.7), then it is an eigenvalue of Problem (1.3) with multiplicity $s+1$ and with linearly independent eigenvectors: $\left(0, w_{1}^{b}\right), \cdots,\left(0, w_{s}^{b}\right),\left(\cos \left(\frac{\pi}{2}+k \pi\right) x_{N}, 0\right)$, for some $k \in \mathbb{N}_{0}$. Roughly speaking, the eigenvalues of Problem (1.3) are obtained by gathering the eigenvalues of Problem (1.7) and the eigenvalues of Problem (1.8). Moreover, each eigenvalue preserves its multiplicity if it is eigenvalue only of Problem (1.7) or only of Problem (1.8); otherwise its multiplicity is obtained by adding the multiplicity as eigenvalue of Problem (1.7) and the multiplicity as eigenvalue of Problem (1.8).

The limit $q$ of the ratio between the volumes of the two cylinders does not intervene in the limit eigenvalue Problem (1.3). It appears in the othonormal conditions:

$$
\begin{gathered}
|\omega| \int_{0}^{1} u_{h}^{a} u_{k}^{a} d x_{N}+q \int_{\omega} u_{h}^{b} u_{k}^{b} d x^{\prime}=\delta_{h, k}, \quad \forall h, k \in \mathbb{N}, \\
\lambda_{h}^{-\frac{1}{2}} \lambda_{k}^{-\frac{1}{2}}\left(|\omega| \int_{0}^{1} u_{h}^{a \prime} u_{k}^{a \prime} d x_{N}+q \int_{\omega} D u_{h}^{b} D u_{k}^{b} d x^{\prime}\right)=\delta_{h, k}, \quad \forall h, k \in \mathbb{N},
\end{gathered}
$$

where $\delta_{h, k}$ is the Kronecker's delta. Moreover, it explicitly intervenes in the corrector result (1.6), and, by the previous othonormal conditions, also in the corrector result (1.5).

If $N=2, q$ appears also in the limit eigenvalue Problem (1.4). More precisely, if $N=2$, the limit problem in $] 0,1[$ is coupled with the limit problem in $\omega$ by the junction conditions:

$$
u^{a}(0)=u^{b}(0) \quad \text { and } \quad|\omega| u^{a \prime}(0)=q\left(u^{b^{\prime}}\left(0^{-}\right)-u^{b^{\prime}}\left(0^{+}\right)\right) .
$$

An easy computation shows that the eigenvalues of Problem (1.4) are given by the positive solutions of the following equation:

$|\omega| \sin (c \sqrt{\lambda}) \sin (d \sqrt{\lambda}) \cos (\sqrt{\lambda})-q \sin (\sqrt{\lambda}) \sin (d \sqrt{\lambda}) \cos (c \sqrt{\lambda})+q \sin (\sqrt{\lambda}) \sin (c \sqrt{\lambda}) \cos (d \sqrt{\lambda})=0$.

For instance, if $\omega=]-1,1\left[\right.$, the set of the eigenvalues of Problem (1.4) is $\left\{\left(k \frac{\pi}{2}\right)^{2}\right\}_{k \in \mathbb{N}}$, and $\left(k \frac{\pi}{2}\right)^{2}$ is a simple eigenvalue if $k$ is odd, it is an eigenvalue with multiplicity 2 if $k$ is even. If $\omega=]-1,2\left[\right.$, the set of the eigenvalues of Problem (1.4) is $\left\{(k \pi)^{2}\right\}_{k \in \mathbb{N}} \cup$ $\left\{\left( \pm \arccos \left( \pm \sqrt{\frac{q}{4 q+2|\omega|}}\right)+2 k \pi\right)^{2}\right\}_{k \in \mathbb{N}_{0}}$.

If $h_{n} \ll r_{n}^{N-1}$, we obtain the following result:

Theorem 1.2. Assume that

$$
\lim _{n} \frac{h_{n}}{r_{n}^{N-1}}=q=0
$$

Then, there exists an increasing diverging sequence of positive numbers $\left\{\lambda_{k}\right\}_{k \in \mathbb{N}}$ satisfying (1.2), and $\left\{\lambda_{k}\right\}_{k \in \mathbb{N}}$ is the set of all the eigenvalues of Problem (1.3) if $N \geq 3$, of the following 
problem:

$$
\left\{\begin{array}{l}
\left.-u^{a \prime \prime}=\lambda u^{a} \text { in }\right] 0,1[, \\
\left.-u^{b^{\prime \prime}}=\lambda u^{b} \text { in }\right] c, 0[, \\
\left.-u^{b^{\prime \prime}}=\lambda u^{b} \text { in }\right] 0, d[, \\
u^{a}(1)=0, \quad u^{a \prime}(0)=0, \\
u^{b}(c)=0, \quad u^{b}(0)=0, \quad u^{b}(d)=0,
\end{array}\right.
$$

if $N=2$ and $\omega=] c, d[$.

There exists an increasing sequence of positive integer numbers $\left\{n_{i}\right\}_{i \in \mathbb{N}}$ and a sequence $\left\{\left(u_{k}^{a}, u_{k}^{b}\right)\right\}_{k \in \mathbb{N}} \subset V_{0}$ (depending possibly on the selected subsequence $\left\{n_{i}\right\}_{i \in \mathbb{N}}$ ), where

$$
V_{0}=\left\{\left(v^{a}, v^{b}\right) \in H^{1}(] 0,1[) \times H^{1}(\omega): v^{a}(1)=0, v^{b}=0 \text { on } \partial \omega,\left(\text { and } v^{b}(0)=0 \text { if } N=2\right)\right\},
$$

satisfying (1.5) and (1.6) with $q=1$, and $u_{k}=\left(u_{k}^{a}, u_{k}^{b}\right)$ is an eigenvector of Problem (1.3) if $N \geq 3$ (Problem (1.11) if $N=2$ ) with eigenvalue $\lambda_{k}$.

Moreover, $\left\{u_{k}\right\}_{k \in \mathbb{N}}$ is a $L^{2}(] 0,1[) \times L^{2}(\omega)$-orthonormal basis with respect to the inner product: $|\omega| \int_{0}^{1} u^{a} v^{a} d x_{N}+\int_{\omega} u^{b} v^{b} d x^{\prime}$, and $\left\{\lambda_{k}^{-\frac{1}{2}} u_{k}\right\}_{k \in \mathbb{N}}$ is a $V_{0}$-Hilbert orthonormal basis with respect to the inner product: $|\omega| \int_{0}^{1} u^{a \prime} v^{a \prime} d x_{N}+\int_{\omega} D u^{b} D v^{b} d x^{\prime}$.

If $N \geq 3$, one obtains the same result as in the previous case when $q=1$ (see Theorem 1.11).

If $N=2$, consider the following problems:

$$
\left\{\begin{array} { l } 
{ - u ^ { a \prime \prime } = \lambda ^ { a } u ^ { a } \text { in } ] 0 , 1 [ , } \\
{ u ^ { a } ( 1 ) = 0 , u ^ { a \prime } ( 0 ) = 0 , }
\end{array} \quad \left\{\begin{array} { l } 
{ - u ^ { b ^ { \prime \prime } } = \lambda ^ { b } u ^ { b } \text { in } ] c , 0 [ , } \\
{ u ^ { b } ( c ) = 0 , u ^ { b } ( 0 ) = 0 , }
\end{array} \quad \left\{\begin{array}{l}
\left.-\bar{u}^{b^{\prime \prime}}=\bar{\lambda}^{b} \bar{u}^{b} \text { in }\right] 0, d[, \\
\bar{u}^{b}(0)=0, \bar{u}^{b}(d)=0 .
\end{array}\right.\right.\right.
$$

Then, with analogous considerations written when $N \geq 3$ after Theorem 1.1. it results that the eigenvalues of Problem (1.11) are obtained by gathering the eigenvalues of these three problems, that is

$$
\left\{\lambda_{k}: k \in \mathbb{N}\right\}=\left\{\left(\frac{\pi}{2}+k \pi\right)^{2}\right\}_{k \in \mathbb{N}_{0}} \cup\left\{\left(\frac{k \pi}{c}\right)^{2}\right\}_{k \in \mathbb{N}} \cup\left\{\left(\frac{k \pi}{d}\right)^{2}\right\}_{k \in \mathbb{N}},
$$

and that the multiplicity of each eigenvalue is less or equal than 3. For instance, if $c=-1$ and $d=1$, the set of the eigenvalues of Problem (1.11) is $\left\{\left(k \frac{\pi}{2}\right)^{2}\right\}_{k \in \mathbb{N}}$, and $\left(k \frac{\pi}{2}\right)^{2}$ is a simple eigenvalue if $k$ is odd, it is an eigenvalue with multiplicity 2 if $k$ is even. If $c=-2$ and $d=2$, the set of the eigenvalues of Problem (1.11) is always $\left\{\left(k \frac{\pi}{2}\right)^{2}\right\}_{k \in \mathbb{N}}$, but $\left(k \frac{\pi}{2}\right)^{2}$ is a simple eigenvalue if $k$ is even, it is an eigenvalue with multiplicity 3 if $k$ is odd.

If $r_{n}^{N-1} \ll h_{n}$ we obtain the following partial result (the result is complete for $N=2$ ) : 
Theorem 1.3. Assume that

$$
\lim _{n} \frac{h_{n}}{r_{n}^{N-1}}=q=+\infty
$$

and

$$
h_{n} \ll-r_{n}^{2} \log r_{n}, \text { if } N=3 ; \quad h_{n} \ll r_{n}^{2}, \text { if } N \geq 4 .
$$

Then, there exists an increasing diverging sequence of positive numbers $\left\{\lambda_{k}\right\}_{k \in \mathbb{N}}$ satisfying (1.2), and $\left\{\lambda_{k}\right\}_{k \in \mathbb{N}}$ is the set of all the eigenvalues of Problem (1.3) if $N \geq 3$, of the following problem:

$$
\left\{\begin{array}{l}
\left.-u^{a \prime \prime}=\lambda u^{a} \text { in }\right] 0,1[, \\
\left.-u^{b^{\prime \prime}}=\lambda u^{b} \text { in } \omega=\right] c, d[, \\
u^{a}(1)=0, \quad u^{a}(0)=0, \\
u^{b}(c)=0, \quad u^{b}(d)=0,
\end{array}\right.
$$

if $N=2$.

There exists an increasing sequence of positive integer numbers $\left\{n_{i}\right\}_{i \in \mathbb{N}}$ and a sequence $\left\{\left(u_{k}^{a}, u_{k}^{b}\right)\right\}_{k \in \mathbb{N}} \subset V_{\infty}$ (depending possibly on the selected subsequence $\left\{n_{i}\right\}_{i \in \mathbb{N}}$ ), where $V_{\infty}=\left\{\left(v^{a}, v^{b}\right) \in H^{1}(] 0,1[) \times H^{1}(\omega): v^{a}(1)=0, v^{b}=0\right.$ on $\partial \omega,\left(\right.$ and $v^{a}(0)=0$ if $\left.\left.N=2\right)\right\}$, satisfying (1.5) and (1.6) with $q=1$, and $u_{k}=\left(u_{k}^{a}, u_{k}^{b}\right)$ is an eigenvector of Problem (1.3) if $N \geq 3$ (Problem 1.14) if $N=2$ ) with eigenvalue $\lambda_{k}$.

Moreover, $\left\{u_{k}\right\}_{k \in \mathbb{N}}$ is a $L^{2}(] 0,1[) \times L^{2}(\omega)$-orthonormal basis with respect to the inner product: $|\omega| \int_{0}^{1} u^{a} v^{a} d x_{N}+\int_{\omega} u^{b} v^{b} d x^{\prime}$, and $\left\{\lambda_{k}^{-\frac{1}{2}} u_{k}\right\}_{k \in \mathbb{N}}$ is a $V_{\infty}$-Hilbert orthonormal basis with respect to the inner product: $|\omega| \int_{0}^{1} u^{a \prime} v^{a \prime} d x_{N}+\int_{\omega} D u^{b} D v^{b} d x^{\prime}$.

If $N \geq 3$, assumption (1.12) and (1.13) reduce to

$$
r_{n}^{2} \ll h_{n} \ll-r_{n}^{2} \log r_{n}, \text { if } N=3 ; \quad r_{n}^{N-1} \ll h_{n} \ll r_{n}^{2}, \text { if } N \geq 4 .
$$

If $N \geq 3$, one obtains the same result as in the previous cases when $q=1$ or $q=0$ (see Theorem 1.1 and Theorem 1.21).

If $N=2$, by considering the following problems:

$$
\left\{\begin{array} { l } 
{ - u ^ { a \prime \prime } = \lambda ^ { a } u ^ { a } \text { in } ] 0 , 1 [ , \quad } \\
{ u ^ { a } ( 1 ) = 0 , \quad u ^ { a } ( 0 ) = 0 , }
\end{array} \quad \left\{\begin{array}{l}
\left.-u^{b^{\prime \prime}}=\lambda^{b} u^{b} \text { in } \omega=\right] c, d[, \\
u^{b}(c)=0, \quad u^{b}(d)=0,
\end{array}\right.\right.
$$

it is possible to repeat similarly the analysis written when $N \geq 3$ after Theorem 1.1. Then, the eigenvalues of Problem (1.14) are obtained by gathering the eigenvalues of these two last problems, that is

$$
\left\{\lambda_{k}: k \in \mathbb{N}\right\}=\left\{(k \pi)^{2}\right\}_{k \in \mathbb{N}} \cup\left\{\left(\frac{k \pi}{d-c}\right)^{2}\right\}_{k \in \mathbb{N}},
$$


and the multiplicity of each eigenvalue is 1 or 2 . For instance, if $] c, d[=]-1,1[$, then the set of all the eigenvalues of Problem (1.14) is $\left\{\left(k \frac{\pi}{2}\right)^{2}\right\}_{k \in \mathbb{N}}$, and $\left(k \frac{\pi}{2}\right)^{2}$ is a simple eigenvalue if $k$ is odd, it is an eigenvalue with multiplicity 2 if $k$ is even. If $] c, d[=]-\frac{1}{2}, \frac{1}{2}[$, then the set of all the eigenvalues of Problem (1.14) is $\left\{(k \pi)^{2}\right\}_{k \in \mathbb{N}}$ and each eigenvalue has multiplicity 2. If $] c, d[=]-\frac{\pi}{2}, \frac{\pi}{2}[$, then the set of all the eigenvalues of Problem (1.14) is $\left\{1,4,9, \pi^{2}, 16,25,36,4 \pi^{2}, 49,64,81,9 \pi^{2}, \cdots\right\}$ and each eigenvalue has multiplicity 1 .

In summarizing:

- the limit eigenvalue problem reduces to a problem in ]0,1[ and a problem in $\omega$ (recall that $\omega$ has dimension dimension $N-1$ ).

- if $N=2$, the limit eigenvalue problem depends on $q$ and it is coupled if $q \in] 0,+\infty[$, uncoupled if $q=0$ or $q=+\infty$. Also the limit eigenvector basis (and the orthonormal limit conditions) depends on $q$.

- If $N \geq 3$ the limit eigenvalue problem is independent of $q$ and it is uncoupled (at least in the considered cases, i.e. for $h_{n} \ll-r_{n}^{2} \log r_{n}$, if $N=3$; for $h_{n} \ll r_{n}^{2}$, if $N \geq 4$ ). The limit eigenvector basis (and the orthonormal limit conditions) depend on $q$. The orthonormal limit conditions coincide for $q \in\{0,1,+\infty\}$.

- If the limit eigenvalue problem is uncoupled (that is when $N=2$ and $q \in\{0,+\infty\}$, or when $N \geq 3$ ), the limit eigenvalues are obtained by gathering the eigenvalues of a Laplace problem in ]0, 1 [ and the eigenvalues of one or two Laplace problems in a domain of dimension $N-1$ and by "adding the multiplicities".

Remark that, in all the previous theorems, by virtue of (1.2), the multiplicity of $\lambda_{n, k}$, for $n$ large enough, is less or equal than the multiplicity of $\lambda_{k}$. Consequently, if $\lambda_{k}$ is simple, also $\lambda_{n, k}$ is simple, for $n$ large enough. Then, by arguing as in [16] (see also [4]), if $\lambda_{k}$ is simple, by fixing one of the two normalized eigenvectors $u_{k}$ of the limit problem with eigenvalue $\lambda_{k}$, it is possible to choose, for $n$ large enough, one of the two normalized eigenvectors $U_{n, k} \in \mathcal{V}_{n}$ of Problem (1.1) with eigenvalue $\lambda_{n, k}$ such that convergences (1.5) and (1.6) hold true for the whole sequence.

Point out that it is not necessary that the two cylinders are scaled to the same one or that the first cylinder has height 1 . In fact, the results do not essentially change if one assumes $\Omega_{n}=\left(r_{n} \omega_{a} \times\left[0, l[) \bigcup\left(\omega_{b} \times\right]-h_{n}, 0[)\right.\right.$, with $0^{\prime} \in \omega^{a} \subset \omega^{b}$ and $\left.l \in\right] 0,+\infty[$.

In Section 2, after having reformulated the problem on a fixed domain through appropriate rescalings of the kind proposed by P.G. Ciarlet and P. Destuynder in [3], and having introduced suitable weighted inner products, by using the min-max Principle we obtain $a$ priori estimates (with respect to $n$ ) for the sequences $\left\{\lambda_{n, k}\right\}_{k \in \mathbb{N}}$ (see Proposition 2.1). Then, by making use of the method of oscillating test functions, introduced by L. Tartar in [17], by applying some results obtained by A. Gaudiello, B. Gustafsson, C. Lefter and J. Mossino in [6] and [7] and by adapting the techniques used by M. Vanninathan in [16], we derive the limit eigenvalue problem and the limit of the rescaled basis, as $n \rightarrow+\infty$, in the case $h_{n} \simeq r_{n}^{N-1}$ (see Theorem [2.5 and the proof of Theorem 1.1] at the end of Section 21). The cases $h_{n} \ll r_{n}^{N-1}$ and $r_{n}^{N-1} \ll h_{n}$ are sketched in Section 3 and Section 4, respectively.

For the study of thin multi-structures we refer to [2], 3], [4], 11], [12, [13, [14] and the 
references quoted therein. For a thin multi-structure as considered in this paper, we refer to [5], [6], [7], [8] [9] and [10. For the study of the spectrum of the Laplace operator in a thin tube with a Dirichlet condition on its boundary we refer to [1. For the study of the homogenization of the spectrum of the Laplace operator in a periodic perforated domain with different boundary conditions on the holes we refer to [16].

2 The case $\left.\lim _{n} \frac{h_{n}}{r_{n}^{N-1}}=q \in\right] 0,+\infty[$

In the sequel, $\left.\Omega^{a}=\omega \times\right] 0,1\left[, \Omega^{b}=\omega \times\right]-1,0\left[\right.$, and $\left\{r_{n}\right\}_{n \in \mathbb{N}}$ and $\left.\left\{h_{n}\right\}_{n \in \mathbb{N}} \subset\right] 0,1[$ are two sequences such that

$$
\lim _{n} h_{n}=0=\lim _{n} r_{n}
$$

For every $n \in \mathbb{N}$, let $H_{n}$ be the space $L^{2}\left(\Omega^{a}\right) \times L^{2}\left(\Omega^{b}\right)$ equipped with the inner product:

$$
\begin{aligned}
& (\cdot, \cdot)_{n}:(u, v)=\left(\left(u^{a}, u^{b}\right),\left(v^{a}, v^{b}\right)\right) \in\left(L^{2}\left(\Omega^{a}\right) \times L^{2}\left(\Omega^{b}\right)\right) \times\left(L^{2}\left(\Omega^{a}\right) \times L^{2}\left(\Omega^{b}\right)\right) \longrightarrow \\
& (u, v)_{n}=\int_{\Omega^{a}} u^{a} v^{a} d x+\frac{h_{n}}{r_{n}^{N-1}} \int_{\Omega^{b}} u^{b} v^{b} d x,
\end{aligned}
$$

and let $V_{n}$ be the space:

$$
\begin{aligned}
& \left\{v=\left(v^{a}, v^{b}\right) \in H^{1}\left(\Omega^{a}\right) \times H^{1}\left(\Omega^{b}\right): v^{a}=0 \text { on } \omega \times\{1\},\right. \\
& \left.v^{b}=0 \text { on } \partial \omega \times\right]-1,0\left[, v^{a}\left(x^{\prime}, 0\right)=v^{b}\left(r_{n} x^{\prime}, 0\right) \text { for } x^{\prime} \text { a.e. in } \omega\right\}
\end{aligned}
$$

equipped with the inner product:

$$
\begin{aligned}
& a_{n}:(u, v)=\left(\left(u^{a}, u^{b}\right),\left(v^{a}, v^{b}\right)\right) \in V_{n} \times V_{n} \longrightarrow a_{n}(u, v)= \\
& \int_{\Omega^{a}} \frac{1}{r_{n}^{2}} D_{x^{\prime}} u^{a} D_{x^{\prime}} v^{a}+\partial_{x_{N}} u^{a} \partial_{x_{N}} v^{a} d x+\frac{h_{n}}{r_{n}^{N-1}} \int_{\Omega^{b}} D_{x^{\prime}} u^{b} D_{x^{\prime}} v^{b}+\frac{1}{h_{n}^{2}} \partial_{x_{N}} u^{b} \partial_{x_{N}} v^{b} d x .
\end{aligned}
$$

The choice of $H_{n}$ and $V_{n}$ will be justified in the proof of Theorem 1.1. at the end of this section. Now, remark that the norm induced on $V_{n}$ by the inner product $a_{n}(\cdot, \cdot)$ is equivalent to the $\left(H^{1}\left(\Omega^{a}\right) \times H^{1}\left(\Omega^{b}\right)\right)$-norm, and the norm induced on $H_{n}$ by the inner product $(\cdot, \cdot)_{n}$ is equivalent to the $\left(L^{2}\left(\Omega^{a}\right) \times L^{2}\left(\Omega^{b}\right)\right)$-norm. Consequently, $V_{n}$ is continuously and compactly embedded into $H_{n}$. Moreover, since $C_{0}^{\infty}\left(\Omega^{a}\right) \times C_{0}^{\infty}\left(\Omega^{b}\right) \subset V_{n}$, it results that $V_{n}$ is dense in $H_{n}$. Then, (for instance, see Th. 6.2-1 and Th. 6.2-2 in [15]) for every $n \in \mathbb{N}$, there exists an increasing diverging sequence of positive numbers $\left\{\lambda_{n, k}\right\}_{k \in \mathbb{N}}$ and a $H_{n}$-Hilbert orthonormal basis $\left\{u_{n, k}\right\}_{k \in \mathbb{N}}$, such that $\left\{\lambda_{n, k}\right\}_{k \in \mathbb{N}}$ forms the set of all the eigenvalues of the following problem:

$$
\left\{\begin{array}{l}
u_{n} \in V_{n}, \\
a_{n}\left(u_{n}, v\right)=\lambda\left(u_{n}, v\right)_{n}, \quad \forall v \in V_{n},
\end{array}\right.
$$


and, for every $k \in \mathbb{N}, u_{n, k} \in V_{n}$ is an eigenvector of (2.5) with eigenvalue $\lambda_{n, k}$. Moreover, $\left\{\lambda_{n, k}^{-\frac{1}{2}} u_{n, k}\right\}_{k \in \mathbb{N}}$ is a $V_{n}$-Hilbert orthonormal basis. Furthermore, for every $k \in \mathbb{N}, \lambda_{n, k}$ is characterized by the following min-max Principle:

$$
\lambda_{n, k}=\min _{\mathcal{E}_{k} \in \mathcal{F}_{k}} \max _{v \in \mathcal{E}_{k}, v \neq 0} \frac{a_{n}(v, v)}{(v, v)_{n}},
$$

where $\mathcal{F}_{k}$ is the set of the subspaces $\mathcal{E}_{k}$ of $V_{n}$ with dimension $k$.

The min-max Principle provides the following a priori estimates for the eigenvalues of Problem (2.5):

Proposition 2.1. For every $n, k \in \mathbb{N}$, let $\lambda_{n, k}$ be as above. Then, it results that

$$
0<\lambda_{n, k} \leq 2^{k} k^{2} \pi^{2}, \quad \forall n, k \in \mathbb{N} .
$$

Proof. It is well known that $\left\{j^{2} \pi^{2}\right\}_{j \in \mathbb{N}}$ is the sequence of the eigenvalues of the following problem:

$$
\left\{\begin{array}{l}
\left.-y^{\prime \prime}(t)=\lambda y(t) \text { in }\right] 0,1[ \\
y(1)=0=y(0)
\end{array}\right.
$$

and $\{\sqrt{2} \sin (j \pi t)\}_{j \in \mathbb{N}}$ is a $L^{2}(] 0,1[)$-Hilbert orthonormal basis such that, for every $j \in \mathbb{N}$, $\sqrt{2} \sin (j \pi t)$ is an eigenvector of (2.8) with eigenvalue $j^{2} \pi^{2}$.

For every $j \in \mathbb{N}$, set

$$
\zeta_{j}\left(x^{\prime}, x_{N}\right)=\left\{\begin{array}{l}
y_{j}\left(x_{N}\right)=\sqrt{2} \sin \left(j \pi x_{N}\right), \text { if }\left(x^{\prime}, x_{N}\right) \in \Omega^{a}, \\
0, \text { if }\left(x^{\prime}, x_{N}\right) \in \Omega^{b}
\end{array}\right.
$$

Fix $k \in \mathbb{N}$ and set $Z_{k}=\left\{\sum_{j=1}^{k} \alpha_{j} \zeta_{j}: \alpha_{1}, \cdots, \alpha_{k} \in \mathbb{R}\right\}$. Then, for every $n \in \mathbb{N}, Z_{k}$ is a subspace of $V_{n}$ of dimension $k$. Consequently, by applying the min-max Principle (2.6), it 
results that:

$$
\begin{gathered}
\lambda_{n, k} \leq \max _{\zeta \in Z_{k}-\{0\}} \frac{a_{n}(\zeta, \zeta)}{(\zeta, \zeta)_{n}}=\max _{\left(\alpha_{1}, \cdots, \alpha_{k}\right) \in \mathbb{R}^{k}-\{0\}} \frac{\int_{0}^{1}\left|\sum_{j=1}^{k} \alpha_{j} y_{j}^{\prime}(t)\right|^{2} d t\left|\sum_{0=1}^{k} \alpha_{j} y_{j}(t)\right|^{2} d t}{\int_{j=1}^{1} d t}= \\
\max _{\left(\alpha_{1}, \cdots, \alpha_{k}\right) \in \mathbb{R}^{k}-\{0\}} \frac{2^{k} \sum_{j=1}^{k} \alpha_{j}^{2} \int_{0}^{1}\left|y_{j}^{\prime}(t)\right|^{2} d t}{\sum_{j=1}^{k} \alpha_{j}^{2}}=\max _{\left(\alpha_{1}, \cdots, \alpha_{k}\right) \in \mathbb{R}^{k}-\{0\}} \frac{2^{k} \sum_{j=1}^{k} \alpha_{j}^{2} j^{2} \pi^{2}}{\sum_{j=1}^{k} \alpha_{j}^{2}} \\
\frac{\max ^{k} k^{2} \pi^{2} \sum_{j=1}^{k} \alpha_{j}^{2}}{\sum_{j=1}^{k} \alpha_{j}^{2}}=2^{k} k^{2} \pi^{2}, \quad \forall n \in \mathbb{N} .
\end{gathered}
$$

Remark 2.2. By examining the previous proof, it is evident that estimate (2.7) holds again true, if one replaces $(\cdot, \cdot)_{n}$ and $a_{n}(\cdot, \cdot)$ with $c_{n}(\cdot, \cdot)_{n}$ and $c_{n} a_{n}(\cdot, \cdot)$, respectively, where $\left.\left\{c_{n}\right\}_{n \in \mathbb{N}} \subset\right] 0,+\infty[$.

By using a diagonal argument, the following result is an immediate consequence of Proposition 2.1]

Corollary 2.3. For every $n, k \in \mathbb{N}$, let $\lambda_{n, k}$ be as above. Then, there exists an increasing sequence of positive integer numbers $\left\{n_{i}\right\}_{i \in \mathbb{N}}$ and an increasing sequence of no negative numbers $\left\{\lambda_{k}\right\}_{k \in \mathbb{N}}$, such that

$$
\lim _{i} \lambda_{n_{i}, k}=\lambda_{k}, \quad \forall k \in \mathbb{N} .
$$

Remark 2.4. In the sequel it will be shown that $\left\{\lambda_{k}\right\}_{k \in \mathbb{N}}$ is a sequence of positive numbers and the convergence holds true for the whole sequence $\left\{\lambda_{n, k}\right\}_{n \in \mathbb{N}}$.

To characterize the sequence $\left\{\lambda_{k}\right\}_{k \in \mathbb{N}}$, consider the following limit:

$$
\lim _{n} \frac{h_{n}}{r_{n}^{N-1}}=q
$$

which exists always for a subsequence. If

$$
q \in] 0,+\infty[
$$


in the space:

$$
H=\left\{v=\left(v^{a}, v^{b}\right) \in L^{2}\left(\Omega^{a}\right) \times L^{2}\left(\Omega^{b}\right): v^{a} \text { is indepenendent of } x^{\prime}, v^{b} \text { is indep. of } x_{N}\right\}
$$

introduce the inner product:

$$
[\cdot, \cdot]_{q}:(u, v)=\left(\left(u^{a}, u^{b}\right),\left(v^{a}, v^{b}\right)\right) \in H \times H \longrightarrow|\omega| \int_{0}^{1} u^{a} v^{a} d x_{N}+q \int_{\omega} u^{b} v^{b} d x^{\prime}
$$

and in the space:

$$
V=\left\{\begin{array}{l}
\left\{v=\left(v^{a}, v^{b}\right) \in H^{1}\left(\Omega^{a}\right) \times H^{1}\left(\Omega^{b}\right):\right. \\
v^{a} \text { is independent of } x^{\prime}, v^{b} \text { is independent of } x_{N}, \\
v^{a}(1)=0, v^{b}=0 \text { on } \partial \omega, \\
\left.v^{a}(0)=v^{b}\left(0^{\prime}\right)\right\}, \\
\left\{v=\left(v^{a}, v^{b}\right) \in H^{1}\left(\Omega^{a}\right) \times H^{1}\left(\Omega^{b}\right):\right. \\
v^{a} \text { is independent of } x^{\prime}, v^{b} \text { is independent of } x_{N}, \\
\left.v^{a}(1)=0, v^{b}=0 \text { on } \partial \omega\right\},
\end{array}\right.
$$

introduce the inner product:

$$
\begin{aligned}
& \alpha_{q}:(u, v)=\left(\left(u^{a}, u^{b}\right),\left(v^{a}, v^{b}\right)\right) \in V \times V \longrightarrow \alpha_{q}(u, v)= \\
& |\omega| \int_{0}^{1} \partial_{x_{N}} u^{a} \partial_{x_{N}} v^{a} d x_{N}+q \int_{\omega} D_{x^{\prime}} u^{b} D_{x^{\prime}} v^{b} d x^{\prime}
\end{aligned}
$$

Remark that the norm induced on $V$ by the inner product $\alpha_{q}(\cdot, \cdot)$ is equivalent to the $\left(H^{1}(] 0,1[) \times H^{1}(\omega)\right)$-norm, and the norm induced on $H$ by the inner product $[\cdot, \cdot]_{q}$ is equivalent to the $\left(L^{2}(] 0,1[) \times L^{2}(\omega)\right)$-norm. Consequently, $V$ is continuously and compactly embedded into $H$. Moreover, since $C_{0}^{\infty}(] 0,1[) \times C_{0}^{\infty}(\omega) \subset V$ if $N \geq 3\left(C_{0}^{\infty}(] 0,1[) \times\{v \in\right.$ $\left.C_{0}^{\infty}(\omega): v(0)=0\right\} \subset V$ if $N=2$ ), it results that $V$ is dense in $H$. Then, one can consider the following eigenvalue problem:

$$
\left\{\begin{array}{l}
u \in V \\
\alpha_{q}(u, v)=\lambda[u, v]_{q}, \quad \forall v \in V
\end{array}\right.
$$

for which all the classic results hold true (see Th. 6.2-1 and 6.2-2 in [15]).

The main result of this paper is the following one: 
Theorem 2.5. For every $n \in \mathbb{N}$, let $\left\{\lambda_{n, k}\right\}_{k \in \mathbb{N}}$ be an increasing diverging sequence of all the eigenvalues of Problem (2.2) $\div$ (2.5), and $\left\{u_{n, k}\right\}_{k \in \mathbb{N}}$ be a $H_{n}$-Hilbert orthonormal basis such that $\left\{\lambda_{n, k}^{-\frac{1}{2}} u_{n, k}\right\}_{k \in \mathbb{N}}$ is a $V_{n}$-Hilbert orthonormal basis and, for every $k \in \mathbb{N}, u_{n, k}$ is an eigenvector of Problem (2.2) $\div$ (2.5) with eigenvalue $\lambda_{n, k}$. Assume (2.1), (2.9) and (2.10).

Then, there exists an increasing diverging sequence of positive numbers $\left\{\lambda_{k}\right\}_{k \in \mathbb{N}}$ such that

$$
\lim _{n} \lambda_{n, k}=\lambda_{k}, \quad \forall k \in \mathbb{N}
$$

and $\left\{\lambda_{k}\right\}_{k \in \mathbb{N}}$ is the set of all the eigenvalues of Problem (2.11) $\div$ 2.15). Moreover, there exists an increasing sequence of positive integer numbers $\left\{n_{i}\right\}_{i \in \mathbb{N}}$ and a $\left(H,[\cdot, \cdot]_{q}\right)$-Hilbert orthonormal basis $\left\{u_{k}\right\}_{k \in \mathbb{N}}$ (depending possibly on the selected subsequence $\left\{n_{i}\right\}_{i \in \mathbb{N}}$ ) such that, for every $k \in \mathbb{N}, u_{k} \in V$ is an eigenvector of Problem (2.11) $\div$ (2.15) with eigenvalue $\lambda_{k}$, and

$$
u_{n_{i}, k} \rightarrow u_{k} \text { strongly in } H^{1}\left(\Omega^{a}\right) \times H^{1}\left(\Omega^{b}\right), \quad \forall k \in \mathbb{N},
$$

as $i \rightarrow+\infty$,

$$
\begin{gathered}
\frac{1}{r_{n}} D_{x^{\prime}} u_{n, k}^{a} \rightarrow 0^{\prime} \text { strongly in }\left(L^{2}\left(\Omega^{a}\right)\right)^{N-1}, \quad \forall k \in \mathbb{N}, \\
\frac{1}{h_{n}} \partial_{x_{N}} u_{n, k}^{b} \rightarrow 0 \text { strongly in } L^{2}\left(\Omega^{b}\right), \quad \forall k \in \mathbb{N},
\end{gathered}
$$

as $n \rightarrow+\infty$. Furthermore, $\left\{\lambda_{k}^{-\frac{1}{2}} u_{k}\right\}_{k \in \mathbb{N}}$ is a $\left(V, \alpha_{q}\right)$-Hilbert orthonormal basis.

Proof. The proof will be performed in three steps.

Step 1. This step is devoted to derive the limit eigenvalue problem.

It results that

$$
\left\{\begin{array}{l}
u_{n, k} \in V_{n}, \\
a_{n}\left(u_{n, k}, v\right)=\lambda_{n, k}\left(u_{n, k}, v\right)_{n}, \quad \forall v \in V_{n}, \quad \forall n, k \in \mathbb{N}, \\
\left(u_{n, k}, u_{n, h}\right)_{n}=\delta_{h, k}, \quad \forall n, k, h, \in \mathbb{N},
\end{array}\right.
$$

where

$$
\delta_{h, k}=\left\{\begin{array}{l}
1, \text { if } h=k, \\
0, \text { if } h \neq k .
\end{array}\right.
$$

By choosing $v=u_{n, k}$ in (2.19), and by taking into account (2.20) and Proposition 2.11 it follows that

$$
a_{n}\left(u_{n, k}, u_{n, k}\right)=\lambda_{n, k}\left(u_{n, k}, u_{n, k}\right)_{n}=\lambda_{n, k} \leq 2^{k} k^{2} \pi^{2}, \quad \forall n, k \in \mathbb{N} .
$$

Consequently, by virtue of definition (2.4) of $a_{n}$ and of assumptions (2.1), (2.9) and (2.10), by applying Proposition 2.1 in [6] (which permits to obtain the junction condition when $N=2$ ) and by using a diagonal argument, it is easily seen that there exists an increasing sequence of positive integer numbers $\left\{n_{i}\right\}_{i \in \mathbb{N}}$, an increasing sequence of no negative numbers $\left\{\lambda_{k}\right\}_{k \in \mathbb{N}}$ 
and a sequence $\left\{u_{k}\right\}_{k \in \mathbb{N}} \subset V$ (depending possibly on the selected subsequence $\left\{n_{i}\right\}_{i \in \mathbb{N}}$ ) such that

$$
\lim _{i} \lambda_{n_{i}, k}=\lambda_{k}, \quad \forall k \in \mathbb{N}
$$

$$
u_{n_{i}, k} \rightarrow u_{k} \text { weakly in } H^{1}\left(\Omega^{a}\right) \times H^{1}\left(\Omega^{b}\right) \text { and strongly in } L^{2}\left(\Omega^{a}\right) \times L^{2}\left(\Omega^{b}\right), \quad \forall k \in \mathbb{N},
$$

as $i \rightarrow+\infty$.

By passing to the limit in (2.19) and (2.20), as $n_{i} \rightarrow+\infty$, by making use of (2.9), (2.10), (2.22), (2.23), and by applying the Dirichlet version of Theorem 1.1 in [7] (see also Remark 1.4 in [7]), with $f=\lambda_{k} u_{k}$, it turns out that $(2.16) \div(2.18)$ hold true and

$$
\begin{cases}u_{k} \in V, & \\ \alpha_{q}\left(u_{k}, v\right)=\lambda_{k}\left[u_{k}, v\right]_{q}, \quad \forall v \in V, & \forall k \in \mathbb{N}, \\ & {\left[u_{k}, u_{h}\right]_{q}=\delta_{h, k}, \quad \forall k, h \in \mathbb{N} .}\end{cases}
$$

In particular, (2.24) and (2.25) provide that

$$
\alpha_{q}\left(\lambda_{k}^{-\frac{1}{2}} u_{k}, \lambda_{h}^{-\frac{1}{2}} u_{h}\right)=\delta_{h, k}, \quad \forall k, h \in \mathbb{N},
$$

Hence, $\left\{\lambda_{k}\right\}_{k \in \mathbb{N}}$ is a sequence of positive numbers. Moreover, remark that

$$
\lim _{k} \lambda_{k}=+\infty
$$

In fact, or (2.26) holds true, or $\left\{\lambda_{k}\right\}_{k \in \mathbb{N}}$ is a finite set. In the second case, by virtue of (2.25), Problem (2.24) would admit an eigenvalue of infinite multiplicity. But this is not possible, due to the Fredholm's alternative Theorem.

Step 2. This step is devoted to prove that there not exist $(\bar{u}, \bar{\lambda}) \in V \times \mathbb{R}$ satisfying the following problem:

$$
\left\{\begin{array}{l}
\bar{u} \in V, \\
\alpha_{q}(\bar{u}, v)=\bar{\lambda}[\bar{u}, v]_{q}, \quad \forall v \in V, \\
{\left[\bar{u}, u_{k}\right]_{q}=0, \quad \forall k \in \mathbb{N}} \\
{[\bar{u}, \bar{u}]_{q}=1 .}
\end{array}\right.
$$

To this aim, the proof of Theorem 9.2 in [16] (see also [4]) will be adapted to our case.

By arguing by contradiction, assume that there exists $(\bar{u}, \bar{\lambda}) \in V \times \mathbb{R}$ satisfying Problem (2.27).

First, remark that (2.26) provides the existence of $\bar{k} \in \mathbb{N}$ such that

$$
\bar{\lambda}<\lambda_{\bar{k}}
$$


For every $n \in \mathbb{N}$, let $\varphi_{n}$ be the solution of the following problem:

$$
\left\{\begin{array}{l}
\varphi_{n} \in V_{n}, \\
a_{n}\left(\varphi_{n}, v\right)=\bar{\lambda}(\bar{u}, v)_{n}, \quad \forall v \in V_{n} .
\end{array}\right.
$$

Then, the Dirichlet version of Theorem 1.1 in [7] (see also Remark 1.4 in [7]), with $f=\bar{\lambda} \bar{u}$, entails the existence of $\varphi \in V$ such that

$$
\varphi_{n} \rightarrow \varphi \text { weakly in } H^{1}\left(\Omega^{a}\right) \times H^{1}\left(\Omega^{b}\right) \text { and strongly in } L^{2}\left(\Omega^{a}\right) \times L^{2}\left(\Omega^{b}\right),
$$

as $n \rightarrow+\infty$, and

$$
\left\{\begin{array}{l}
\varphi \in V, \\
\alpha_{q}(\varphi, v)=\bar{\lambda}[\bar{u}, v]_{q}, \quad \forall v \in V .
\end{array}\right.
$$

By comparing (2.27) with (2.31) and by virtue of the uniqueness of $\varphi$, it turns out that

$$
\varphi=\bar{u} \text {. }
$$

For every $n \in \mathbb{N}$, set

$$
v_{n}=\varphi_{n}-\sum_{i=1}^{\bar{k}}\left(\varphi_{n}, u_{n, i}\right)_{n} u_{n, i} \in V_{n} .
$$

Then, by virtue of (2.20), it results that

$$
\begin{array}{r}
\left(v_{n}, u_{n, k}\right)_{n}=\left(\varphi_{n}, u_{n, k}\right)_{n}-\sum_{i=1}^{\bar{k}}\left(\varphi_{n}, u_{n, i}\right)_{n}\left(u_{n, i}, u_{n, k}\right)_{n}=\left(\varphi_{n}, u_{n, k}\right)_{n}-\left(\varphi_{n}, u_{n, k}\right)_{n}=0 \\
\forall k \in\{1, \cdots, \bar{k}\}, \quad \forall n \in \mathbb{N} .
\end{array}
$$

Consequently, by recalling that $\left\{\lambda_{n, k}^{-\frac{1}{2}} u_{n, k}\right\}_{k \in \mathbb{N}}$ is a $V_{n}$-Hilbert orthonormal basis, that $u_{n, k}$ solves Problem (2.19), that $\left\{\lambda_{n, k}\right\}_{k \in \mathbb{N}}$ is an increasing sequence and that $\left\{u_{n, k}\right\}_{k \in \mathbb{N}}$ is a $H_{n^{-}}$ Hilbert orthonormal basis, it follows that

$$
\begin{aligned}
& a_{n}\left(v_{n}, v_{n}\right)=\sum_{k=1}^{+\infty}\left|a_{n}\left(\lambda_{n, k}^{-\frac{1}{2}} u_{n, k}, v_{n}\right)\right|^{2}=\sum_{k=1}^{+\infty} \lambda_{n, k}^{-1} \lambda_{n, k}^{2}\left(u_{n, k}, v_{n}\right)_{n}^{2}= \\
& \sum_{k=\bar{k}+1}^{+\infty} \lambda_{n, k}\left(u_{n, k}, v_{n}\right)_{n}^{2} \geq \lambda_{n, \bar{k}+1} \sum_{k=\bar{k}+1}^{+\infty}\left(u_{n, k}, v_{n}\right)_{n}^{2}=\lambda_{n, \bar{k}+1}\left(v_{n}, v_{n}\right)_{n}, \quad \forall n \in \mathbb{N} .
\end{aligned}
$$

In what concerns the first term in (2.33),

$$
\begin{aligned}
& a_{n}\left(v_{n}, v_{n}\right)=a_{n}\left(\varphi_{n}-\sum_{k=1}^{\bar{k}}\left(\varphi_{n}, u_{n, k}\right)_{n} u_{n, k}, \varphi_{n}-\sum_{j=1}^{\bar{k}}\left(\varphi_{n}, u_{n, j}\right)_{n} u_{n, j}\right)= \\
& a_{n}\left(\varphi_{n}, \varphi_{n}\right)-2 \sum_{k=1}^{\bar{k}}\left(\varphi_{n}, u_{n, k}\right)_{n} a_{n}\left(\varphi_{n}, u_{n, k}\right)+\sum_{k, j=1}^{\bar{k}}\left(\varphi_{n}, u_{n, k}\right)_{n}\left(\varphi_{n}, u_{n, j}\right)_{n} a_{n}\left(u_{n, k}, u_{n, j}\right), \quad \forall n \in \mathbb{N} .
\end{aligned}
$$


from which, by virtue of (2.29), (2.19) and (2.20), it follows that

$$
\begin{aligned}
& a_{n}\left(v_{n}, v_{n}\right)=\bar{\lambda}\left(\bar{u}, \varphi_{n}\right)_{n}-2 \bar{\lambda} \sum_{k=1}^{\bar{k}}\left(\varphi_{n}, u_{n, k}\right)_{n}\left(\bar{u}, u_{n, k}\right)_{n}+\sum_{k, j=1}^{\bar{k}}\left(\varphi_{n}, u_{n, k}\right)_{n}\left(\varphi_{n}, u_{n, j}\right)_{n} \lambda_{n, k}\left(u_{n, k}, u_{n, j}\right)_{n}= \\
& \bar{\lambda}\left(\bar{u}, \varphi_{n}\right)_{n}-2 \bar{\lambda} \sum_{k=1}^{\bar{k}}\left(\varphi_{n}, u_{n, k}\right)_{n}\left(\bar{u}, u_{n, k}\right)_{n}+\sum_{k=1}^{\bar{k}}\left(\varphi_{n}, u_{n, k}\right)_{n}^{2} \lambda_{n, k}, \quad \forall n \in \mathbb{N} .
\end{aligned}
$$

Consequently, by virtue of (2.9), (2.10), (2.30), (2.32), (2.16), (2.22) and (2.27), it results that

$$
\lim _{i} a_{n_{i}}\left(v_{n_{i}}, v_{n_{i}}\right)=\bar{\lambda}[\bar{u}, \bar{u}]_{q}-2 \bar{\lambda} \sum_{k=1}^{\bar{k}}\left[\bar{u}, u_{k}\right]_{q}^{2}+\sum_{k=1}^{\bar{k}}\left[\bar{u}, u_{k}\right]_{q}^{2} \lambda_{k}=\bar{\lambda} .
$$

In what concerns the last term in (2.33), equalities (2.20) provide that

$$
\begin{aligned}
& \left(v_{n}, v_{n}\right)_{n}=\left(\varphi_{n}-\sum_{k=1}^{\bar{k}}\left(\varphi_{n}, u_{n, k}\right)_{n} u_{n, k}, \varphi_{n}-\sum_{j=1}^{\bar{k}}\left(\varphi_{n}, u_{n, j}\right)_{n} u_{n, j}\right)_{n}= \\
& \left(\varphi_{n}, \varphi_{n}\right)_{n}-2 \sum_{k=1}^{\bar{k}}\left(\varphi_{n}, u_{n, k}\right)_{n}\left(\varphi_{n}, u_{n, k}\right)_{n}+\sum_{k, j=1}^{\bar{k}}\left(\varphi_{n}, u_{n, k}\right)_{n}\left(\varphi_{n}, u_{n, j}\right)_{n}\left(u_{n, k}, u_{n, j}\right)_{n}= \\
& \left(\varphi_{n}, \varphi_{n}\right)_{n}-2 \sum_{k=1}^{\bar{k}}\left(\varphi_{n}, u_{n, k}\right)_{n}^{2}+\sum_{k=1}^{\bar{k}}\left(\varphi_{n}, u_{n, k}\right)_{n}^{2},=\left(\varphi_{n}, \varphi_{n}\right)_{n}-\sum_{k=1}^{\bar{k}}\left(\varphi_{n}, u_{n, k}\right)_{n}^{2}, \quad \forall n \in \mathbb{N} .
\end{aligned}
$$

Consequently, by virtue of (2.9), (2.10), (2.30), (2.32), (2.16), (2.27), it results that

$$
\lim _{i}\left(v_{n_{i}}, v_{n_{i}}\right)_{n_{i}}=[\bar{u}, \bar{u}]_{q}-\sum_{k=1}^{\bar{k}}\left[\bar{u}, u_{k}\right]_{q}^{2}=1 .
$$

Finally, by passing to the limit in (2.33) as $n_{i} \rightarrow+\infty$, convergences (2.34), (2.22) and (2.35) entail that

$$
\bar{\lambda} \geq \lambda_{\bar{k}}
$$

which is in contradiction with (2.28). Hence, Problem (2.27) does not admit solution. Step 3. Conclusion.

In Step 1, it is proved that $\left.\left\{\lambda_{k}\right\}_{k \in \mathbb{N}} \subset\right] 0,+\infty[$ is an increasing and diverging sequence of eigenvalues of Problem (2.24),$\left\{u_{k}\right\}_{k \in \mathbb{N}}$ is an orthonormal sequence in $\left(H,[\cdot, \cdot]_{q}\right),\left\{\lambda_{k}^{-\frac{1}{2}} u_{k}\right\}_{k \in \mathbb{N}}$ is an orthonormal sequence in $\left(V, \alpha_{q}\right)$, and, for every $k \in \mathbb{N}, u_{k}$ is an eigenvector for Problem (2.24), with eigenvalue $\lambda_{k}$.

Indeed, the sequence $\left\{\lambda_{k}\right\}_{k \in \mathbb{N}}$ forms the whole set of the eigenvalues of Problem (2.24). In fact, if $\bar{\lambda} \notin\left\{\lambda_{k}\right\}_{k \in \mathbb{N}}$ is another eigenvalue of Problem (2.24) and $\bar{u} \in V$ is a corresponding eigenvector (which can be assumed $H$-normalized), it results that

$$
\bar{\lambda}\left[\bar{u}, u_{k}\right]_{q}=\alpha_{q}\left(\bar{u}, u_{k}\right)=\alpha_{q}\left(u_{k}, \bar{u}\right)=\lambda_{k}\left[u_{k}, \bar{u}\right]_{q}, \quad \forall k \in \mathbb{N},
$$


that is

$$
\left[\bar{u}, u_{k}\right]_{q}=0, \quad \forall k \in \mathbb{N},
$$

which is in contradiction with the statement of Step 2.

It remains to prove that the set of the finite combinations of elements of $\left\{\lambda_{k}^{-\frac{1}{2}} u_{k}\right\}_{k \in \mathbb{N}}$ is dense in $\left(V, \alpha_{q}\right)$, which provides that the set of the finite combinations of elements of $\left\{u_{k}\right\}_{k \in \mathbb{N}}$ is dense in $\left(H,[\cdot, \cdot]_{q}\right)$, since $V$ is continuously embedded into $H$, with dense inclusion.

It is well known that $\left(V, \alpha_{q}\right)$ has a Hilbert orthonormal basis of eigenvectors of Problem (2.15) (for instance, see Th. 6.2-1 in [15]). Consequently, by denoting with $\left\{\mu_{i}\right\}_{i \in \mathbb{N}}$ the sequence of all different eigenvalues of Problem (2.15) (i.e. $\mu_{i} \neq \mu_{j}$ for $i \neq j$ ) and with $\left\{E_{i}\right\}_{i \in \mathbb{N}}$ the sequence of the spaces of the associated eigenvectors, it results that the vectorial space generated by $\left\{E_{i}\right\}_{i \in \mathbb{N}}$ is dense in $\left(V, \alpha_{q}\right)$. Recall that, for every $i \in \mathbb{N}, E_{i}$ has finite dimension and $E_{i}$ is orthogonal to $E_{j}$ (with respect to $\alpha_{q}$ ) if $i \neq j$. Then, to conclude it is enough to show that, for every $i \in \mathbb{N}$, an orthonormal basis (with respect to $\alpha_{q}$ ) of $E_{i}$ is included in $\left\{\lambda_{k}^{-\frac{1}{2}} u_{k}\right\}_{k \in \mathbb{N}}$. This last property will be proved by arguing by contradiction. If it is not true, there exist $\bar{i} \in \mathbb{N}$ and $\bar{u} \in E_{\bar{i}}$ such that $\alpha_{q}\left(\mu_{\bar{i}}^{-\frac{1}{2}} \bar{u}, u_{k}\right)=0$ for every $k \in \mathbb{N}$, and $\alpha_{q}\left(\mu_{\bar{i}}^{-\frac{1}{2}} \bar{u}, \mu_{\bar{i}}^{-\frac{1}{2}} \bar{u}\right)=1$. That is $\bar{u}$ is an eigenvector of Problem (2.15), with eigenvalue $\mu_{\bar{i}}$, such that $\left[\bar{u}, u_{k}\right]_{q}=0$ for every $k \in \mathbb{N}$, and $[\bar{u}, \bar{u}]_{q}=1$, in contradiction with the statement of Step 2 .

In conclusion, since the sequence $\left\{\lambda_{k}\right\}_{k \in \mathbb{N}}$ can be characterized by the min-max Principle (for instance, see Th. 6.2-2 in [15]), for every $k \in \mathbb{N}$, convergence (2.22) holds true for the whole sequence $\left\{\lambda_{n, k}\right\}_{n \in \mathbb{N}}$.

Proof of Theorem 1.1. As it is usual (see [3]), Problem (1.1) can be reformulated on a fixed domain through an appropriate rescaling which maps $\Omega_{n}$ into $\left.\Omega=\omega \times\right]-1,1$. Namely, by setting

$$
\widetilde{u}_{n, k}(x)=\left\{\begin{array}{ll}
\widetilde{u}_{n, k}^{a}\left(x^{\prime}, x_{3}\right)=U_{n, k}\left(r_{n} x^{\prime}, x_{3}\right), & \left.\left(x^{\prime}, x_{3}\right) \text { a.e. in } \Omega^{a}=\omega \times\right] 0,1[, \quad \\
\widetilde{u}_{n, k}^{b}\left(x^{\prime}, x_{3}\right)=U_{n, k}\left(x^{\prime}, h_{n} x_{3}\right), & \left.\left(x^{\prime}, x_{3}\right) \text { a.e. in } \Omega^{b}=\omega \times\right]-1,0[
\end{array} \quad \forall n, k \in \mathbb{N},\right.
$$

it results that, for every $n \in \mathbb{N},\left\{\lambda_{n, k}\right\}_{k \in \mathbb{N}}$ forms the set of all the eigenvalues of Problem (2.5), $u_{n, k}=r_{n}^{\frac{N-1}{2}} \widetilde{u}_{n, k} \in V_{n}$ is an eigenvector of (2.5) with eigenvalue $\lambda_{n, k},\left\{u_{n, k}\right\}_{k \in \mathbb{N}}$ is a $H_{n}$-Hilbert orthonormal basis and $\left\{\lambda_{n, k}^{-\frac{1}{2}} u_{n, k}\right\}_{k \in \mathbb{N}}$ is a $V_{n}$-Hilbert orthonormal basis, where $V_{n}$ and $H_{n}$ are defined at the beginning of this section. Then, Theorem 1.1 is an immediate consequence of Theorem 2.5 .

3 The case $\lim _{n} \frac{h_{n}}{r_{n}^{N-1}}=0$

For every $n, k \in \mathbb{N}$, let $\lambda_{n, k}$ be as in Section 2. The aim of this section is to investigate the limit behavior, as $n \rightarrow+\infty$, of $\left\{\lambda_{n, k}\right\}_{n \in \mathbb{N}}$, under the assumption:

$$
\lim _{n} \frac{h_{n}}{r_{n}^{N-1}}=0
$$


Let $H_{0}$ be the space $H$ given in (2.11) equipped with the inner product $[\cdot, \cdot]_{1}$ defined by (2.12) with $q=1$. Moreover, let

$$
V_{0 b}= \begin{cases}\left\{v^{b} \in H^{1}\left(\Omega^{b}\right): v^{b} \text { is independent of } x_{N}, v^{b}=0 \text { on } \partial \omega,\right. & \text { if } N=2 ; \\ \left.v^{b}\left(0^{\prime}\right)=0\right\}, & \\ \left\{v^{b} \in H^{1}\left(\Omega^{b}\right): v^{b} \text { is independent of } x_{N}, v^{b}=0 \text { on } \partial \omega\right\}, & \text { if } 3 \leq N\end{cases}
$$

equipped with the $H_{0}^{1}(\omega)$-norm, and $V_{0}$ be the space

$$
V_{0}=\left\{v^{a} \in H^{1}\left(\Omega^{a}\right): v^{a} \text { is independent of } x^{\prime}, v^{a}(1)=0\right\} \times V_{0 b}
$$

equipped with the inner product $\alpha_{1}$ defined by (2.14) with $q=1$. Remark that $V_{0}$ is continuously and compactly embedded into $H_{0}$, with dense inclusion.

Let

$$
\widetilde{V}_{0 b}=\left\{v^{b} \in W_{0}^{1, \infty}(\omega): v^{b}=0 \text { in a neighbourhood of } 0^{\prime}\right\}
$$

then the following density result holds true:

Proposition 3.1. $\widetilde{V}_{0 b}$ is dense in $V_{0 b}$.

Proof. We sketch the proof.

The density result is obvious if $N=2$.

Assume $N>2$ and let $\left\{\varepsilon_{n}\right\}_{n \in \mathbb{N}} \subset \mathbb{R},\left\{\eta_{n}\right\}_{n \in \mathbb{N}} \subset \mathbb{R}$ and $\left\{\varphi_{n}\right\}_{n \in \mathbb{N}} \subset C\left(\mathbb{R}^{N-1}\right)$ three sequences such that, for every $n \in \mathbb{N}, 0<\varepsilon_{n}<\eta_{n}<\operatorname{dist}\left(0^{\prime}, \partial \omega\right), \varphi_{n}=1$ in $\left\{x^{\prime} \in \mathbb{R}^{N-1}\right.$ : $\left.\left|x^{\prime}\right| \leq \varepsilon_{n}\right\}, \varphi_{n}=0$ in $\mathbb{R}^{N-1}-\left\{x^{\prime} \in \mathbb{R}^{N-1}:\left|x^{\prime}\right|<\eta_{n}\right\},\left\{\varphi_{n}\right\}_{n \in \mathbb{N}} \subset C^{1}\left(\left\{x^{\prime} \in \mathbb{R}^{N-1}: \varepsilon_{n} \leq\left|x^{\prime}\right| \leq\right.\right.$ $\left.\left.\eta_{n}\right\}\right), 0 \leq \varphi_{n} \leq 1$, and $\lim _{n} \eta_{n}=0=\lim _{n} \int_{\left\{x^{\prime} \in \mathbb{R}^{N-1}: \varepsilon_{n}<\left|x^{\prime}\right|<\eta_{n}\right\}}\left|D \varphi_{n}\right|^{2} d x^{\prime}$ (for the existence of such sequences, see Proposition 3.1 in [6]).

Since $C_{0}^{\infty}(\omega)$ is dense in $V_{0 b}$, it is enough to prove that $\widetilde{V}_{0 b}$ is dense in $C_{0}^{\infty}(\omega)$ with respect to the $H_{0}^{1}(\omega)$-norm. For $v \in C_{0}^{\infty}(\omega)$, set $v_{n}=\left(1-\varphi_{n}\right) v \in \widetilde{V}_{0 b}$ for every $n \in \mathbb{N}$. Since $v=\varphi_{n} v+\left(1-\varphi_{n}\right) v$ for every $n \in \mathbb{N}$, and

$$
\begin{aligned}
& \lim _{n} \int_{\omega}\left|D\left(v \varphi_{n}\right)\right|^{2} d x^{\prime} \leq \\
& \|v\|_{W^{1, \infty}(\omega)}^{2} \lim _{n}\left(\operatorname{meas}\left\{x^{\prime} \in \mathbb{R}^{N-1}:\left|x^{\prime}\right|<\eta_{n}\right\}+\int_{\left\{x^{\prime} \in \mathbb{R}^{N-1}: \varepsilon_{n}<\left|x^{\prime}\right|<\eta_{n}\right\}}\left|D \varphi_{n}\right|^{2} d x^{\prime}\right)=0,
\end{aligned}
$$

it follows that $v_{n} \rightarrow v$ strongly in $H_{0}^{1}(\omega)$.

Consider the eigenvalue problem:

$$
\left\{\begin{array}{l}
u \in V_{0}, \\
\alpha_{1}(u, v)=\lambda[u, v]_{1}, \quad \forall v \in V_{0},
\end{array}\right.
$$

then, if (3.1) holds true, the following convergence result yields: 
Theorem 3.2. For every $n \in \mathbb{N}$, let $\left\{\lambda_{n, k}\right\}_{k \in \mathbb{N}}$ be an increasing diverging sequence of all the eigenvalues of Problem (2.2) $\div$ (2.5), and $\left\{\bar{u}_{n, k}\right\}_{k \in \mathbb{N}}$ be a Hilbert orthonormal basis for the space $H_{n}$ equipped with the inner product: $\frac{r_{n}^{N-1}}{h_{n}}(\cdot, \cdot)_{n}$, such that $\left\{\lambda_{n, k}^{-\frac{1}{2}} \bar{u}_{n, k}\right\}_{k \in \mathbb{N}}$ is a Hilbert orthonormal basis for the space $V_{n}$ equipped with the inner product $\frac{r_{n}^{N-1}}{h_{n}} a_{n}(\cdot, \cdot)$ and, for every $k \in \mathbb{N}, \bar{u}_{n, k}$ is an eigenvector of Problem (2.2) $\div$ (2.5) with eigenvalue $\lambda_{n, k}$. Assume (2.1) and (3.1).

Then, there exists an increasing diverging sequence of positive numbers $\left\{\lambda_{k}\right\}_{k \in \mathbb{N}}$ such that

$$
\lim _{n} \lambda_{n, k}=\lambda_{k}, \quad \forall k \in \mathbb{N},
$$

and $\left\{\lambda_{k}\right\}_{k \in \mathbb{N}}$ is the set of all the eigenvalues of Problem (3.2), (3.3), (3.5). Moreover, there exists an increasing sequence of positive integer numbers $\left\{n_{i}\right\}_{i \in \mathbb{N}}$ and an $H_{0}$-Hilbert orthonormal basis $\left\{u_{k}=\left(u_{k}^{a}, u_{k}^{b}\right)\right\}_{k \in \mathbb{N}}$ (depending possibly on the selected subsequence $\left\{n_{i}\right\}_{i \in \mathbb{N}}$ ) such that, for every $k \in \mathbb{N}, u_{k} \in V_{0}$ is an eigenvector of Problem (3.2), (3.3), (3.5), with eigenvalue $\lambda_{k}$, and

$$
\frac{r_{n_{i}}^{\frac{N-1}{2}}}{h_{n_{i}}^{\frac{1}{2}}} \bar{u}_{n_{i}, k}^{a} \rightarrow u_{k}^{a} \text { strongly in } H^{1}\left(\Omega^{a}\right), \quad \bar{u}_{n_{i}, k}^{b} \rightarrow u_{k}^{b}, \text { strongly in } H^{1}\left(\Omega^{b}\right), \quad \forall k \in \mathbb{N},
$$

as $i \rightarrow+\infty$,

$$
\left\{\begin{array}{l}
\frac{1}{r_{n}} \frac{r_{n}^{\frac{N-1}{2}}}{h_{n}^{\frac{1}{2}}} D_{x^{\prime}} \bar{u}_{n, k}^{a} \rightarrow 0^{\prime} \text { strongly in }\left(L^{2}\left(\Omega^{a}\right)\right)^{N-1}, \\
\frac{1}{h_{n}} \partial_{x_{N}} \bar{u}_{n, k}^{b} \rightarrow 0 \text { strongly in } L^{2}\left(\Omega^{b}\right),
\end{array} \quad \forall k \in \mathbb{N},\right.
$$

as $n \rightarrow+\infty$. Furthermore, $\left\{\lambda_{k}^{-\frac{1}{2}} u_{k}\right\}_{k \in \mathbb{N}}$ is a $V_{0}$-Hilbert orthonormal basis.

Proof. We sketch the proof.

By multiplying (2.5) by $\frac{r_{n}^{N-1}}{h_{n}}$, it results that

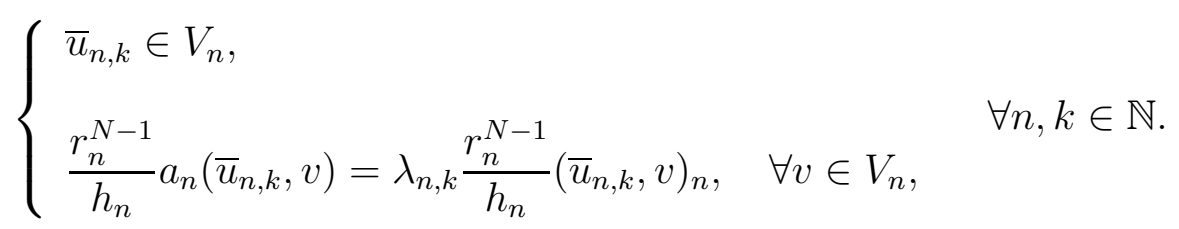

Moreover, since $\left\{\bar{u}_{n, k}\right\}_{k \in \mathbb{N}}$ is a Hilbert orthonormal basis for the space $H_{n}$ equipped with the inner product $\frac{r_{n}^{N-1}}{h_{n}}(\cdot, \cdot)_{n}$, one has that

$$
\frac{r_{n}^{N-1}}{h_{n}}\left(\bar{u}_{n, k}, \bar{u}_{n, h}\right)_{n}=\delta_{h, k}, \quad \forall n, k, h, \in \mathbb{N},
$$


where $\delta_{h, k}$ is defined in (2.21)

By choosing $v=\bar{u}_{n, k}$ in (3.8), and by taking into account (3.9), Proposition 2.1 and Remark 2.2, it follows that

$$
\frac{r_{n}^{N-1}}{h_{n}} a_{n}\left(\bar{u}_{n, k}, \bar{u}_{n, k}\right)=\lambda_{n, k} \frac{r_{n}^{N-1}}{h_{n}}\left(\bar{u}_{n, k}, \bar{u}_{n, k}\right)_{n}=\lambda_{n, k} \leq 2^{k} k^{2} \pi^{2}, \quad \forall n, k \in \mathbb{N} .
$$

Consequently, by virtue of definition (2.4) of $a_{n}$ and of assumption (2.1), and by using a diagonal argument, it is easily seen that there exists an increasing sequence of positive integer numbers $\left\{n_{i}\right\}_{i \in \mathbb{N}}$, an increasing sequence of no negative numbers $\left\{\lambda_{k}\right\}_{k \in \mathbb{N}}$, a sequence $\left\{u_{k}=\left(u_{k}^{a}, u_{k}^{b}\right)\right\}_{k \in \mathbb{N}} \subset H^{1}\left(\Omega^{a}\right) \times H^{1}\left(\Omega^{b}\right)$ (depending possibly on the selected subsequence $\left.\left\{n_{i}\right\}_{i \in \mathbb{N}}\right)$, with $u_{k}^{a}$ independent of $x^{\prime}, u_{k}^{b}$ independent of $x_{N}, u_{k}^{a}(1)=0, u_{k}^{b}=0$ on $\partial \omega$, and a sequence $\left\{\left(\xi_{k}^{a}, \xi_{k}^{b}\right)\right\}_{k \in \mathbb{N}} \subset\left(L^{2}\left(\Omega^{a}\right)\right)^{N-1} \times L^{2}\left(\Omega^{b}\right)$ (depending possibly on the selected subsequence $\left.\left\{n_{i}\right\}_{i \in \mathbb{N}}\right)$ such that

$$
\lim _{i} \lambda_{n_{i}, k}=\lambda_{k}, \quad \forall k \in \mathbb{N},
$$

$$
\begin{aligned}
& \left\{\begin{array}{l}
\frac{r_{n_{i}}^{\frac{N-1}{2}}}{h_{n_{i}}^{\frac{1}{2}}} \bar{u}_{n_{i}, k}^{a} \rightarrow u_{k}^{a} \text { weakly in } H^{1}\left(\Omega^{a}\right) \text { and strongly in } L^{2}\left(\Omega^{a}\right), \\
\bar{u}_{n_{i}, k}^{b} \rightarrow u_{k}^{b} \text { weakly in } H^{1}\left(\Omega^{b}\right) \text { and strongly in } L^{2}\left(\Omega^{b}\right),
\end{array} \quad \forall k \in \mathbb{N},\right. \\
& \left\{\begin{array}{l}
\frac{r_{n_{i}}^{\frac{N-1}{2}}}{h_{n_{i}}^{\frac{1}{2}}} \frac{1}{r_{n_{i}}} D_{x^{\prime}} \bar{u}_{n_{i}, k}^{a}-\xi_{k}^{a} \text { weakly in }\left(L^{2}\left(\Omega^{a}\right)\right)^{N-1}, \\
\frac{1}{h_{n_{i}}} \partial_{x_{N}} \bar{u}_{n_{i}, k}^{b} \rightarrow \xi_{k}^{b} \text { weakly in } L^{2}\left(\Omega^{b}\right),
\end{array} \quad \forall k \in \mathbb{N},\right.
\end{aligned}
$$

as $i \rightarrow+\infty$.

To obtain $u^{k} \in V_{0}$, for every $k \in \mathbb{N}$, it remains to prove that

$$
u_{k}^{b}\left(0^{\prime}\right)=0, \quad \forall k \in \mathbb{N}, \quad \text { if } N=2 .
$$

Indeed, statement (2.4) in [6] (which holds true also if $q=0$ !) gets

$$
\lim _{i} \int_{\omega} \bar{u}_{n_{i}, k}^{b}\left(r_{n_{i}} x^{\prime}, 0\right) d x^{\prime}=|\omega| u_{k}^{b}\left(0^{\prime}\right), \quad \forall k \in \mathbb{N}, \quad \text { if } N=2 .
$$

Then, by combining (3.14) with the first line in (3.11), and by taking into account that $u_{n_{i}, k}^{a}\left(x^{\prime}, 0\right)=u_{n_{i}, k}^{b}\left(r_{n_{i}} x^{\prime}, 0\right)$ for $x^{\prime}$ a.e. in $\omega$ and (3.1) , one obtains that

$$
\begin{aligned}
& |\omega| u_{k}^{b}\left(0^{\prime}\right)=\lim _{i} \int_{\omega} \bar{u}_{n_{i}, k}^{b}\left(r_{n_{i}} x^{\prime}, 0\right) d x^{\prime}=\lim _{i} \int_{\omega} \bar{u}_{n_{i}, k}^{a}\left(x^{\prime}, 0\right) d x^{\prime}= \\
& \lim _{i}\left(\frac{h_{n_{i}}^{\frac{1}{2}}}{r_{n_{i}}^{\frac{N-1}{2}}} \int_{\omega} \frac{r_{n_{i}}^{\frac{N-1}{2}}}{h_{n_{i}}^{\frac{1}{2}}} \bar{u}_{n_{i}, k}^{a}\left(x^{\prime}, 0\right) d x^{\prime}\right)=0|\omega| u_{k}^{a}(0)=0, \quad \forall k \in \mathbb{N}, \quad \text { if } N=2,
\end{aligned}
$$


that is (3.13).

Now, by passing to the limit, as $n_{i} \rightarrow+\infty$, in (3.8), with $v=\left\{\begin{array}{ll}0 & \text { in } \Omega^{a}, \\ v^{b} & \text { in } \Omega^{b}\end{array}\right.$ and $v^{b} \in \widetilde{V}_{0 b}$, and by making use of (3.10) and of the second line of (3.11), it turns out that

$$
\int_{\omega} D_{x^{\prime}} u_{k}^{b} D_{x^{\prime}} v^{b} d x^{\prime}=\lambda_{k} \int_{\omega} u_{k}^{b} v^{b} d x^{\prime}, \quad \forall v^{b} \in \widetilde{V}_{0 b}, \quad \forall k \in \mathbb{N},
$$

and, consequently, by virtue of Proposition 3.1

$$
\int_{\omega} D_{x^{\prime}} u_{k}^{b} D_{x^{\prime}} v^{b} d x^{\prime}=\lambda_{k} \int_{\omega} u_{k}^{b} v^{b} d x^{\prime}, \quad \forall v^{b} \in V_{0 b}, \quad \forall k \in \mathbb{N} .
$$

On the other hand, by passing to the limit, as $n_{i} \rightarrow+\infty$, in

$$
\frac{r_{n}^{\frac{N-1}{2}}}{h_{n}^{\frac{1}{2}}} a_{n}\left(\bar{u}_{n, k}, v\right)=\lambda_{n, k} \frac{r_{n}^{\frac{N-1}{2}}}{h_{n}^{\frac{1}{2}}}\left(\bar{u}_{n, k}, v\right)_{n}, \quad \forall v \in V_{n}, \quad \forall k \in \mathbb{N},
$$

with $v=\left\{\begin{array}{ll}v^{a} & \text { in } \Omega^{a}, \\ v^{b} & \text { in } \Omega^{b}\end{array}\right.$ and $v^{a} \in H^{1}(] 0,1[), v^{a}(1)=0, v^{b} \in C_{0}^{1}(\omega), v^{b}=v^{a}(0)$ in a neighbourhood of $0^{\prime}$, and by making use of (3.10) and of (3.11), it turns out that

$$
|\omega| \int_{0}^{1} \partial_{x_{N}} u_{k}^{a} \partial_{x_{N}} v^{a} d x_{N}=\lambda_{k}|\omega| \int_{0}^{1} u_{k}^{a} v^{a} d x_{N}, \forall v^{a} \in H^{1}(] 0,1[): v^{a}(1)=0, \forall k \in \mathbb{N} .
$$

By adding (3.15) to (3.16), one obtains that

$$
\left\{\begin{array}{l}
u_{k} \in V_{0}, \\
\alpha_{1}\left(u_{k}, v\right)=\lambda_{k}\left[u_{k}, v\right]_{1}, \quad \forall v \in V_{0},
\end{array} \quad \forall k \in \mathbb{N} .\right.
$$

Finally, by passing to the limit, as $n_{i} \rightarrow+\infty$, in (3.9), and by making use of (3.11) and (3.17), it turns out that

$$
\alpha_{1}\left(\lambda_{k}^{-\frac{1}{2}} u_{k}, \lambda_{h}^{-\frac{1}{2}} u_{h}\right)=\left[u_{k}, u_{h}\right]_{1}=\delta_{h, k}, \quad \forall k, h \in \mathbb{N} .
$$

Moreover, by arguing as in the proof of Theorem [2.5, one proves that

$$
\lim _{k} \lambda_{k}=+\infty
$$

Now, let us identify $\xi_{k}^{a}$ and $\xi_{k}^{b}$. By choosing $v=\bar{u}_{n, k}$ as test function in (3.8), by replacing $n$ with $n_{i}$, by passing to the liminf as $i$ diverges, by taking into account of (3.10), (3.11), (3.12) and (3.18), and by using a l.s.c. argument it results that

$$
\int_{\Omega^{a}}\left|\xi_{k}^{a}\right|^{2}+\left|\partial_{x_{N}} u_{k}^{a}\right|^{2} d x+\int_{\Omega^{b}}\left|D_{x^{\prime}} u_{k}^{b}\right|^{2}+\left|\xi_{k}^{b}\right|^{2} d x \leq \int_{\Omega^{a}}\left|\partial_{x_{N}} u_{k}^{a}\right|^{2} d x+\int_{\Omega^{b}}\left|D_{x^{\prime}} u_{k}^{b}\right|^{2} d x, \forall k \in \mathbb{N},
$$

which provides that

$$
\xi_{k}^{a}=0, \quad \xi_{k}^{b}=0, \quad \forall k \in \mathbb{N}
$$


The strong convergences in (3.6) and (3.7) follows from (3.11), (3.12), (3.19) and from the convergence of the energies:

$$
\lim _{i} \frac{r_{n_{i}}^{N-1}}{h_{n_{i}}} a_{n_{i}}\left(\bar{u}_{n_{i}, k}, \bar{u}_{n_{i}, k}\right)=\lim _{i} \lambda_{n_{i}, k}=\lambda_{k}=\alpha_{1}\left(u_{k}, u_{k}\right), \quad \forall k \in \mathbb{N} .
$$

As in Step 2 of the proof of Theorem 2.5, by arguing by contradiction, one can show that there not exist $(\bar{u}, \bar{\lambda}) \in V_{0} \times \mathbb{R}$ satisfying the following problem:

$$
\left\{\begin{array}{l}
\bar{u} \in V_{0}, \\
\alpha_{1}(\bar{u}, v)=\bar{\lambda}[\bar{u}, v]_{1}, \quad \forall v \in V_{0}, \\
{\left[\bar{u}, u_{k}\right]_{1}=0, \quad \forall k \in \mathbb{N}} \\
{[\bar{u}, \bar{u}]_{1}=1 .}
\end{array}\right.
$$

Precisely, assume that there exists $(\bar{u}, \bar{\lambda}) \in V_{0} \times \mathbb{R}$ satisfying (3.21). Let $\bar{k} \in \mathbb{N}$ be such that

$$
\bar{\lambda}<\lambda_{\bar{k}}
$$

and, for every $n \in \mathbb{N}$, let $\varphi_{n}$ be the solution of the following problem:

$$
\left\{\begin{array}{l}
\varphi_{n} \in V_{n}, \\
a_{n}\left(\varphi_{n}, v\right)=\bar{\lambda}\left(\left(\frac{h_{n}^{\frac{1}{2}}}{r_{n}^{\frac{N-1}{2}}} \bar{u}^{a}, \bar{u}^{b}\right), v\right)_{n}, \quad \forall v \in V_{n}
\end{array}\right.
$$

where $\bar{u}=\left(\bar{u}^{a}, \bar{u}^{b}\right)$. Then, it is easy to prove that

$$
\frac{r_{n}^{\frac{N-1}{2}}}{h_{n}^{\frac{1}{2}}} \varphi_{n}^{a} \rightarrow \bar{u}^{a} \text { weakly in } H^{1}\left(\Omega^{a}\right), \quad \varphi_{n}^{b} \rightarrow \bar{u}^{b} \text { weakly in } H^{1}\left(\Omega^{b}\right),
$$

as $n \rightarrow+\infty$. Moreover, for every $n \in \mathbb{N}$, set

$$
v_{n}=\varphi_{n}-\sum_{i=1}^{\bar{k}} \frac{r_{n}^{N-1}}{h_{n}}\left(\varphi_{n}, u_{n, i}\right)_{n} u_{n, i} \in V_{n}
$$

Then, by proceeding as in the proof of Step 2 of Theorem 2.5, but by taking care to replace $(\cdot, \cdot)_{n}$ with $\frac{r_{n}^{N-1}}{h_{n}}(\cdot, \cdot)_{n}$ and $a_{n}(\cdot, \cdot)$ with $\frac{r_{n}^{N-1}}{h_{n}} a_{n}(\cdot, \cdot)$, one reaches a contradiction.

To complete the proof, one can argue as Step 3 of the proof of Theorem 2.5.

Remark 3.3. For every $n \in \mathbb{N}$, let $\left\{\lambda_{n, k}\right\}_{k \in \mathbb{N}}$ be an increasing diverging sequence of all the eigenvalues of Problem (2.2) $\div$ (2.5), and $\left\{u_{n, k}\right\}_{k \in \mathbb{N}}$ be a Hilbert orthonormal basis for the space $H_{n}$, such that $\left\{\lambda_{n, k}^{-\frac{1}{2}} u_{n, k}\right\}_{k \in \mathbb{N}}$ is a Hilbert orthonormal basis for the space $V_{n}$, and, for every $k \in \mathbb{N}, u_{n, k}$ is an eigenvector of (2.5) with eigenvalue $\lambda_{n, k}$. 
By setting $\bar{u}_{n, k}=\frac{h_{n}^{\frac{1}{2}}}{r_{n}^{\frac{N-1}{2}}} u_{n, k}$, it turns out that, for every $n \in \mathbb{N},\left\{\bar{u}_{n, k}\right\}_{k \in \mathbb{N}}$ is a Hilbert orthonormal basis for the space $H_{n}$ equipped with the inner product $\frac{r_{n}^{N-1}}{h_{n}}(\cdot, \cdot)_{n},\left\{\lambda_{n, k}^{-\frac{1}{2}} \bar{u}_{n, k}\right\}_{k \in \mathbb{N}}$ is a Hilbert orthonormal basis for the space $V_{n}$ equipped with the inner product $\frac{r_{n}^{N-1}}{h_{n}} a_{n}(\cdot, \cdot)$, and, for every $k \in \mathbb{N}, \bar{u}_{n, k}$ is an eigenvector of (2.5) with eigenvalue $\lambda_{n, k}$.

Then, by applying Theorem [3.2, it follows that

$$
\lim _{n} \lambda_{n, k}=\lambda_{k}, \quad \forall k \in \mathbb{N}
$$

$$
u_{n_{i}, k}^{a} \rightarrow u_{k}^{a} \text { strongly in } H^{1}\left(\Omega^{a}\right), \quad \frac{h_{n_{i}}^{\frac{1}{2}}}{r_{n_{i}}^{\frac{N-1}{2}}} u_{n_{i}, k}^{b} \rightarrow u_{k}^{b}, \text { strongly in } H^{1}\left(\Omega^{b}\right), \quad \forall k \in \mathbb{N},
$$

as $i \rightarrow+\infty$,

$\frac{1}{r_{n}} D_{x^{\prime}} u_{n, k}^{a} \rightarrow 0^{\prime}$ strongly in $\left(L^{2}\left(\Omega^{a}\right)\right)^{N-1}, \frac{1}{h_{n}^{\frac{1}{2}} r_{n}^{\frac{N-1}{2}}} \partial_{x_{N}} u_{n, k}^{b} \rightarrow 0$, strongly in $L^{2}\left(\Omega^{b}\right), \forall k \in \mathbb{N}$, as $n \rightarrow+\infty$, where $\left\{\lambda_{k}\right\}_{k \in \mathbb{N}}$ and $\left\{\left(u_{k}^{a}, u_{k}^{b}\right)\right\}_{k \in \mathbb{N}}$ are given by Theorem 3.2.

Proof of Theorem 1.2. Theorem 1.2 follows immediately from Remark 3.3, by arguing as in the proof of Theorem 1.1 at the end of Section 2.

\section{The case $\lim _{n} \frac{h_{n}}{r_{n}^{N-1}}=+\infty$}

For every $n, k \in \mathbb{N}$, let $\lambda_{n, k}$ be as in Section [2] The aim of this section is to investigate the asymptotic behavior, as $n \rightarrow+\infty$, of $\left\{\lambda_{n, k}\right\}_{n \in \mathbb{N}}$, under the assumption:

$$
\lim _{n} \frac{h_{n}}{r_{n}^{N-1}}=+\infty
$$

Indeed, in this case, only a partial result is obtained. Precisely, for $N=2$, the limit eigenvalue problem is completely derived; while, for $N \geq 3$, it is obtained only under the additional assumption:

$$
\left\{\begin{array}{l}
h_{n} \ll-r_{n}^{2} \log r_{n}, \text { if } N=3, \\
h_{n} \ll r_{n}^{2}, \text { if } N \geq 4 .
\end{array}\right.
$$

As in Section 3, let $H_{0}$ be the space $H$ given in (2.11) equipped with the inner product 
$[\cdot, \cdot]_{1}$ defined by (2.12) with $q=1$. Moreover, let $V_{\infty}$ be the space:

$$
V_{\infty}=\left\{\begin{array}{l}
\left\{v=\left(v^{a}, v^{b}\right) \in H^{1}\left(\Omega^{a}\right) \times H^{1}\left(\Omega^{b}\right):\right. \\
v^{a} \text { is independent of } x^{\prime}, v^{b} \text { is independent of } x_{N}, \\
v^{a}(1)=0, v^{a}(0)=0, \\
\left.v^{b}=0 \text { on } \partial \omega\right\}, \\
\left\{v=\left(v^{a}, v^{b}\right) \in H^{1}\left(\Omega^{a}\right) \times H^{1}\left(\Omega^{b}\right):\right. \\
v^{a} \text { is independent of } x^{\prime}, v^{b} \text { is independent of } x_{N}, \\
\left.v^{a}(1)=0, v^{b}=0 \text { on } \partial \omega\right\},
\end{array} \text { if } 3 \leq N ;\right.
$$

equipped with the inner product $\alpha_{1}$ defined by (2.14) with $q=1$. Remark that $V_{\infty}$ is continuously and compactly embedded into $H_{0}$, with dense inclusion.

Consider the following eigenvalue problem:

$$
\left\{\begin{array}{l}
u \in V_{\infty} \\
\alpha_{1}(u, v)=\lambda[u, v]_{1}, \quad \forall v \in V_{\infty}
\end{array}\right.
$$

then, if (4.1) and (4.2) hold true, the following convergence result yields:

Theorem 4.1. For every $n \in \mathbb{N}$, let $\left\{\lambda_{n, k}\right\}_{k \in \mathbb{N}}$ be an increasing diverging sequence of all the eigenvalues of Problem (2.2) $\div$ (2.5), and $\left\{u_{n, k}\right\}_{k \in \mathbb{N}}$ be a $H_{n}$-Hilbert orthonormal basis such that $\left\{\lambda_{n, k}^{-\frac{1}{2}} u_{n, k}\right\}_{k \in \mathbb{N}}$ is a $V_{n}$-Hilbert orthonormal basis and, for every $k \in \mathbb{N}, u_{n, k}$ is an eigenvector of Problem (2.2) $\div$ (2.5) with eigenvalue $\lambda_{n, k}$. Assume (2.1), (4.1) and, for $N \geq 3$, also (4.2).

Then, there exists an increasing diverging sequence of positive numbers $\left\{\lambda_{k}\right\}_{k \in \mathbb{N}}$ such that

$$
\lim _{n} \lambda_{n, k}=\lambda_{k}, \quad \forall k \in \mathbb{N}
$$

and $\left\{\lambda_{k}\right\}_{k \in \mathbb{N}}$ is the set of all the eigenvalues of Problem 4.3), (4.4). Moreover, there exists an increasing sequence of positive integer numbers $\left\{n_{i}\right\}_{i \in \mathbb{N}}$ and an $H_{0}$-Hilbert orthonormal basis $\left\{u_{k}=\left(u_{k}^{a}, u_{k}^{b}\right)\right\}_{k \in \mathbb{N}}$ (depending possibly on the selected subsequence $\left\{n_{i}\right\}_{i \in \mathbb{N}}$ ) such that, for every $k \in \mathbb{N}, u_{k} \in V_{\infty}$ is an eigenvector of Problem (4.3), (4.4), with eigenvalue $\lambda_{k}$, and

$$
u_{n_{i}, k}^{a} \rightarrow u_{k}^{a} \text { strongly in } H^{1}\left(\Omega^{a}\right), \quad \frac{h_{n_{i}}^{\frac{1}{2}}}{r_{n_{i}}^{\frac{N-1}{2}}} u_{n_{i}, k}^{b} \rightarrow u_{k}^{b}, \text { strongly in } H^{1}\left(\Omega^{b}\right), \quad \forall k \in \mathbb{N},
$$


as $i \rightarrow+\infty$.

$$
\left\{\begin{array}{l}
\frac{1}{r_{n}} D_{x^{\prime}} u_{n, k}^{a} \rightarrow 0^{\prime} \text { strongly in }\left(L^{2}\left(\Omega^{a}\right)\right)^{N-1}, \\
\frac{1}{h_{n}} \frac{h_{n}^{\frac{1}{2}}}{r_{n}^{\frac{N-1}{2}}} \partial_{x_{N}} u_{n, k}^{b} \rightarrow 0 \text { strongly in } L^{2}\left(\Omega^{b}\right),
\end{array} \quad \forall k \in \mathbb{N},\right.
$$

as $n \rightarrow+\infty$. Furthermore, $\left\{\lambda_{k}^{-\frac{1}{2}} u_{k}\right\}_{k \in \mathbb{N}}$ is a $V_{\infty}$-Hilbert orthonormal basis.

Proof. We sketch the proof.

It results that

$$
\begin{aligned}
& \left\{\begin{array}{l}
u_{n, k} \in V_{n}, \\
a_{n}\left(u_{n, k}, v\right)=\lambda_{n, k}\left(u_{n, k}, v\right)_{n}, \quad \forall v \in V_{n},
\end{array} \quad \forall n, k \in \mathbb{N},\right. \\
& \left(u_{n, k}, u_{n, h}\right)_{n}=\delta_{h, k}, \quad \forall n, k, h, \in \mathbb{N},
\end{aligned}
$$

where $\delta_{h, k}$ is defined in (2.21).

By choosing $v=u_{n, k}$ in (4.7), and by taking into account (4.8) and Proposition 2.1, it follows that

$$
a_{n}\left(u_{n, k}, u_{n, k}\right)=\lambda_{n, k}\left(u_{n, k}, u_{n, k}\right)_{n}=\lambda_{n, k} \leq 2^{k} k^{2} \pi^{2}, \quad \forall n, k \in \mathbb{N} .
$$

Consequently, by virtue of definition (2.4) of $a_{n}$ and of assumption (2.1), and by using a diagonal argument, it is easily seen that there exists an increasing sequence of positive integer numbers $\left\{n_{i}\right\}_{i \in \mathbb{N}}$, an increasing sequence of no negative numbers $\left\{\lambda_{k}\right\}_{k \in \mathbb{N}}$, a sequence $\left\{u_{k}=\left(u_{k}^{a}, u_{k}^{b}\right)\right\}_{k \in \mathbb{N}} \subset H^{1}\left(\Omega^{a}\right) \times H^{1}\left(\Omega^{b}\right)$ (depending possibly on the selected subsequence $\left.\left\{n_{i}\right\}_{i \in \mathbb{N}}\right)$, with $u_{k}^{a}$ independent of $x^{\prime}, u_{k}^{b}$ independent of $x_{N}, u_{k}^{a}(1)=0, u_{k}^{b}=0$ on $\partial \omega$, and a sequence $\left\{\left(\xi_{k}^{a}, \xi_{k}^{b}\right)\right\}_{k \in \mathbb{N}} \subset\left(L^{2}\left(\Omega^{a}\right)\right)^{N-1} \times L^{2}\left(\Omega^{b}\right)$ (depending possibly on the selected subsequence $\left.\left\{n_{i}\right\}_{i \in \mathbb{N}}\right)$ such that

$$
\begin{gathered}
\lim _{i} \lambda_{n_{i}, k}=\lambda_{k}, \quad \forall k \in \mathbb{N}, \\
\left\{\begin{array}{l}
u_{n_{i}, k}^{a} \rightarrow u_{k}^{a} \text { weakly in } H^{1}\left(\Omega^{a}\right) \text { and strongly in } L^{2}\left(\Omega^{a}\right), \\
\frac{h_{n_{i}}^{\frac{1}{2}}}{r_{n_{i}}^{\frac{N-1}{2}}} u_{n_{i}, k}^{b} \rightarrow u_{k}^{b} \text { weakly in } H^{1}\left(\Omega^{b}\right) \text { and strongly in } L^{2}\left(\Omega^{b}\right),
\end{array}\right. \\
\left\{\begin{array}{l}
\frac{1}{r_{n_{i}}} D_{x^{\prime}} u_{n_{i}, k}^{a} \rightarrow \xi_{k}^{a} \text { weakly in }\left(L^{2}\left(\Omega^{a}\right)\right)^{N-1}, \\
\frac{1}{h_{n_{i}}} \frac{h_{n_{n_{i}}}^{\frac{N-1}{2}}}{r_{n_{N}}} \partial_{x_{N}} u_{n_{i}, k}^{b} \rightarrow \xi_{k}^{b} \text { weakly in } L^{2}\left(\Omega^{b}\right),
\end{array}\right.
\end{gathered}
$$

as $i \rightarrow+\infty$. 
To obtain $u^{k} \in V_{\infty}$, for every $k \in \mathbb{N}$, it remains to prove that

$$
u_{k}^{a}(0)=0, \quad \forall k \in \mathbb{N}, \quad \text { if } N=2 .
$$

By virtue of the first line in (4.11) and of the fact that $u_{n_{i}, k}^{a}\left(x^{\prime}, 0\right)=u_{n_{i}, k}^{b}\left(r_{n_{i}} x^{\prime}, 0\right)$ for $x^{\prime}$ a.e. in $\omega$, for obtaining (4.13), it is enough to prove that

$$
\lim _{i} \int_{\omega} u_{n_{i}, k}^{b}\left(r_{n_{i}} x^{\prime}, 0\right) d x^{\prime}=0, \quad \forall k \in \mathbb{N}
$$

By taking into account that $u_{n, k}^{b} \rightarrow 0$ strongly in $H^{1}\left(\Omega^{b}\right)$ and that $\left\|\frac{\partial u_{n, k}^{b}}{\partial x_{N}}\right\|_{L^{2}\left(\Omega^{b}\right)} \leq c r_{n}^{\frac{N-1}{2}} h_{n}^{\frac{1}{2}}$, by adapting the the proof of (2.4) in [6], limit (4.14) can be achieved, if $N=2$.

By choosing in (4.7), written with $n=n_{i}$,

$$
v= \begin{cases}0 & \text { in } \omega \times] \varepsilon_{i}, 1[, \\ \frac{r_{n_{i}}^{\frac{N-1}{2}}}{h_{n_{i}}^{\frac{1}{2}}} v^{b}\left(r_{n_{i}} x^{\prime}\right) \frac{\varepsilon_{i}-x_{N}}{\varepsilon_{i}} & \text { in } \omega \times] 0, \varepsilon_{i}[, \\ \frac{r_{n_{i}}^{\frac{N-1}{2}}}{h_{n_{i}}^{\frac{1}{2}}} v^{b} & \text { in } \Omega^{b},\end{cases}
$$

with $v^{b} \in C_{0}^{\infty}(\omega)$ and $\left.\left\{\varepsilon_{i}\right\}_{i \in \mathbb{N}} \subset\right] 0,1[$, it results that

$$
\begin{aligned}
& \frac{r_{n_{i}}^{\frac{N-1}{2}}}{h_{n_{i}}^{\frac{1}{2}}} \int_{\omega} \int_{0}^{\varepsilon_{i}} \frac{1}{r_{n_{i}}} D_{x^{\prime}} u_{n_{i}, k}^{a}\left(D_{x^{\prime}} v^{b}\right)\left(r_{n_{i}} x^{\prime}\right) \frac{\varepsilon_{i}-x_{N}}{\varepsilon_{i}}-\partial_{x_{N}} u_{n_{i}, k}^{a} v^{b}\left(r_{n_{i}} x^{\prime}\right) \frac{1}{\varepsilon_{i}} d x+\int_{\Omega^{b}} \frac{h_{n_{i}}^{\frac{1}{2}}}{r_{n_{i}}^{\frac{N-1}{2}}} D_{x^{\prime}} u_{n_{i}, k}^{b} D_{x^{\prime}} v^{b} d x \\
& =\lambda_{n_{i}, k} \frac{r_{n_{i}}^{\frac{N-1}{2}}}{h_{n_{i}}^{\frac{1}{2}}} \int_{\omega} \int_{0}^{\varepsilon_{i}} u_{n_{i}, k}^{a} v^{b}\left(r_{n_{i}} x^{\prime}\right) \frac{\varepsilon_{i}-x_{N}}{\varepsilon_{i}} d x+\lambda_{n_{i}, k} \int_{\Omega^{b}} \frac{h_{n_{i}}^{\frac{1}{2}}}{r_{n_{i}}^{\frac{N-1}{2}}} u_{n_{i}, k}^{b} v^{b} d x, \forall i \in \mathbb{N}, \quad \forall k \in \mathbb{N} .
\end{aligned}
$$

Consequently, by passing to the limit as $i \rightarrow+\infty$, and by making use of (4.1) and (4.10) $\div$ (4.11), one achieves

$$
\int_{\omega} D_{x^{\prime}} u_{k}^{b} D_{x^{\prime}} v^{b} d x^{\prime}=\lambda_{k} \int_{\omega} u_{k}^{b} v^{b} d x^{\prime}, \quad \forall v^{b} \in H_{0}^{1}(\omega), \quad \forall k \in \mathbb{N}
$$

if the sequence $\left\{\varepsilon_{i}\right\}_{i \in \mathbb{N}}$ has been chosen such that $\lim _{i} \varepsilon_{i}=0$ and $\frac{r_{n_{i}}^{N-1}}{h_{n_{i}}}<<\varepsilon_{i}$.

Assume now $N=2$.

By passing to the limit, as $n_{i} \rightarrow+\infty$, in (4.7), with $v=\left\{\begin{array}{ll}v^{a} & \text { in } \Omega^{a}, \\ 0 & \text { in } \Omega^{b}\end{array}\right.$ and $v^{a} \in$ $H_{0}^{1}(] 0,1[)$, and by making use of (4.10) and of the first line of (4.11), it turns out that

$$
|\omega| \int_{0}^{1} \partial_{x_{N}} u_{k}^{a} \partial_{x_{N}} v^{a} d x_{N}=\lambda_{k}|\omega| \int_{0}^{1} u_{k}^{a} v^{a} d x_{N}, \quad \forall v^{a} \in H_{0}^{1}(] 0,1[), \quad \forall k \in \mathbb{N} .
$$


By adding (4.15) to (4.16), one obtains that

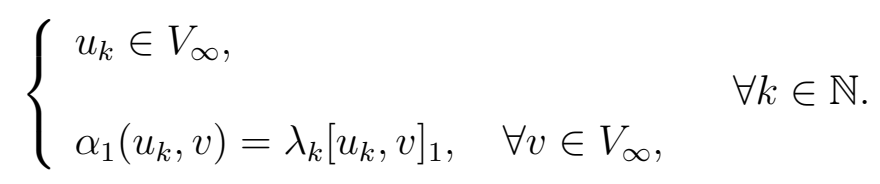

if $N=2$. Finally, by passing to the limit, as $n_{i} \rightarrow+\infty$, in (4.8), and by making use of (4.11) and (4.17), it turns out that

$$
\alpha_{1}\left(\lambda_{k}^{-\frac{1}{2}} u_{k}, \lambda_{h}^{-\frac{1}{2}} u_{h}\right)=\left[u_{k}, u_{h}\right]_{1}=\delta_{h, k}, \quad \forall k, h \in \mathbb{N} .
$$

Moreover, by arguing as in the proof of Theorem 2.5, one proves that

$$
\lim _{k} \lambda_{k}=+\infty
$$

Now, assume $N \geq 4$.

Let $\left\{\varphi_{n}\right\}_{n \in \mathbb{N}} \subset C\left(\mathbb{R}^{N-1}\right)$ be a sequence such that, for every $n \in \mathbb{N}, \varphi_{n}=1$ in $\left\{x^{\prime} \in\right.$ $\left.\mathbb{R}^{N-1}:\left|x^{\prime}\right| \leq r_{n}\right\}, \varphi_{n}=0$ in $\mathbb{R}^{N-1}-\left\{x^{\prime} \in \mathbb{R}^{N-1}:\left|x^{\prime}\right|<2 r_{n}\right\},\left\{\varphi_{n}\right\}_{n \in \mathbb{N}} \subset C^{1}\left(\left\{x^{\prime} \in \mathbb{R}^{N-1}:\right.\right.$ $\left.\left.r_{n} \leq\left|x^{\prime}\right| \leq 2 r_{n}\right\}\right), 0 \leq \varphi_{n} \leq 1, \int_{\left\{x^{\prime} \in \mathbb{R}^{N-1}: r_{n}<\left|x^{\prime}\right|<2 r_{n}\right\}}\left|D \varphi_{n}\right|^{2} d x^{\prime}=c r_{n}^{N-3}$, where $c$ is a constant independent of $n$, but dependent on $N-1$ (for the existence of such sequence, see Proposition 3.1 in [6] $)$.

Now, by passing to the limit, as $n_{i} \rightarrow+\infty$, in (4.7), with $v= \begin{cases}v^{a} & \text { in } \Omega^{a}, \\ v^{a}\left(0^{\prime}\right) \varphi_{n}\left(\frac{x^{\prime}}{d}\right) & \text { in } \Omega^{b}\end{cases}$ and $v^{a} \in H^{1}(] 0,1[), v^{a}(1)=0$, where $d=\operatorname{dist}\left(0^{\prime}, \partial \omega\right)$, and by taking into account the additional assumption (4.2), it is easily seen that

$$
|\omega| \int_{0}^{1} \partial_{x_{N}} u_{k}^{a} \partial_{x_{N}} v^{a} d x_{N}=|\omega| \lambda_{k} \int_{0}^{1} u_{k}^{a} v^{a} d x_{N}, \quad \forall v^{a} \in H^{1}(] 0,1[), \quad \forall k \in \mathbb{N} .
$$

By adding (4.20) to (4.15), one obtains (4.17) (and consequently (4.18) and (4.19)), if $4 \leq N$ and $r_{n}^{N-1} \ll h_{n} \ll r_{n}^{2}$.

If $N=3$, one obtains (4.20) by arguing as above, but by choosing $\varphi_{n}$ such that, for every $n \in \mathbb{N}, \varphi_{n}=1$ in $\left\{x^{\prime} \in \mathbb{R}^{N-1}:\left|x^{\prime}\right| \leq r_{n}\right\}, \varphi_{n}=0$ in $\mathbb{R}^{N-1}-\left\{x^{\prime} \in \mathbb{R}^{N-1}:\left|x^{\prime}\right|<\sqrt{r_{n}}\right\}$, $\left\{\varphi_{n}\right\}_{n \in \mathbb{N}} \subset C^{1}\left(\left\{x^{\prime} \in \mathbb{R}^{N-1}: r_{n} \leq\left|x^{\prime}\right| \leq \sqrt{r_{n}}\right\}\right), 0 \leq \varphi_{n} \leq 1, \int_{\left\{x^{\prime} \in \mathbb{R}^{N-1}: r_{n}<\left|x^{\prime}\right|<\sqrt{r_{n}}\right\}}\left|D \varphi_{n}\right|^{2} d x^{\prime}=$ $-c\left(\log r_{n}\right)^{-1}$, where $\mathrm{c}$ is a positive constant (for the existence of such sequence, see Proposition 3.1 in [6]), and $r^{2} \ll h_{n} \ll-r_{n}^{2} \log r_{n}$.

The identification of $u_{k}^{a}$ remains an open question when $N \geq 3$ and assumption (4.2) is not satisfied.

Let, now, $N=2$, or $N=3$ and $r_{n}^{2} \ll h_{n} \ll-r_{n}^{2} \log r_{n}$, or $N \geq 4$ and $r_{n}^{N-1} \ll h_{n} \ll r_{n}^{2}$. As in Step 2 of the proof of Theorem 2.5, by arguing by contradiction, one can show that 
there not exist $(\bar{u}, \bar{\lambda}) \in V_{\infty} \times \mathbb{R}$ satisfying the following problem:

$$
\left\{\begin{array}{l}
\bar{u} \in V_{\infty}, \\
\alpha_{1}(\bar{u}, v)=\bar{\lambda}[\bar{u}, v]_{1}, \quad \forall v \in V_{\infty}, \\
{\left[\bar{u}, u_{k}\right]_{1}=0, \quad \forall k \in \mathbb{N}} \\
{[\bar{u}, \bar{u}]_{1}=1 .}
\end{array}\right.
$$

Precisely, assume that there exists $(\bar{u}, \bar{\lambda}) \in V_{\infty} \times \mathbb{R}$ satisfying (4.21). Let $\bar{k} \in \mathbb{N}$ be such that

$$
\bar{\lambda}<\lambda_{\bar{k}}
$$

and, for every $n \in \mathbb{N}$, let $\varphi_{n}$ be the solution of the following problem:

$$
\left\{\begin{array}{l}
\varphi_{n} \in V_{n} \\
a_{n}\left(\varphi_{n}, v\right)=\bar{\lambda}\left(\left(\bar{u}^{a}, \frac{r_{n}^{\frac{N-1}{2}}}{h_{n}^{\frac{1}{2}}} \bar{u}^{b}\right), v\right)_{n}, \quad \forall v \in V_{n}
\end{array}\right.
$$

where $\bar{u}=\left(\bar{u}^{a}, \bar{u}^{b}\right)$. Then, it is easy to prove that

$$
\varphi_{n}^{a} \rightarrow \bar{u}^{a} \text { weakly in } H^{1}\left(\Omega^{a}\right), \quad \frac{h_{n}^{\frac{1}{2}}}{r_{n}^{\frac{N-1}{2}}} \varphi_{n}^{b} \rightarrow \bar{u}^{b} \text { weakly in } H^{1}\left(\Omega^{b}\right),
$$

as $n \rightarrow+\infty$. Then, by proceeding as in the proof of Step 2 of Theorem 2.5, one reaches a contradiction.

To complete the proof, one can argue as Step 3 of the proof of Theorem 2.5.

Proof of Theorem 1.3. Theorem 1.3 follows immediately from Theorem 4.1, by arguing as in the proof of Theorem 1.1 at the end of Section 2 .

\section{Acknowledgments}

The authors wish to thank "LATP" of the University of Provence (Aix-Marseille I), where the first author was invited as visiting professor in May 2005 and in May 2006 and where this research has been initiated and concluded.

This paper is part of the project: "Strutture sottili" of the program 2004-2006: "Collaborazioni interuniversitarie internazionali" of the Italian Ministry of Education, University and Research. 


\section{References}

[1] G. Bouchitté, L. Mascarenhas, L. Trabucho, On the Curvature and Torsion Effects in One Dimensional Waveguides, Preprint n. 2006 -02 of Lab. d'Analyse non Linéaire Appliquée et Modelisation, University of Sud Toulon-Var.

[2] P.G. Ciarlet, Plates and Junctions in Elastic Multistructures: An Asymptotic Analysis. Research in Applied Mathematics, 14. Masson, Paris; Springer-Verlag, Berlin, (1990).

[3] P.G. Ciarlet, P. Destuynder, A Justification of the Two-Dimensional Linear Plate Model, J. Mècanique 18 (1979), n.2, 315-344.

[4] D. Cioranescu, J. Saint Jean Paulin, Homogenization of Reticulated Structures. Applied Mathematical Sciences, 139, Springer-Verlag, New York.

[5] A. Gaudiello, B. Gustafsson, C. Lefter, J. Mossino, Coupled and Uncoupled Limits for a N-Dimensional Multidomain Neumann Problem, C. R. Acad. Sci. Paris Sér. I Math. 330 (2000), 985-990.

[6] A. Gaudiello, B. Gustafsson, C. Lefter, J. Mossino, Asymptotic Analysis of a Class of Minimization Problems in a Thin Multidomain, Calc. Var. Partial Differential Equations 15 (2002), n.2, 181-201.

[7] A. Gaudiello, B. Gustafsson, C. Lefter, J. Mossino, Asymptotic Analysis for Monotone Quasilinear Problems in Thin Multidomains, Differential Integral Equations 15 (2002), n.5, 623-640.

[8] A. Gaudiello, R. Monneau, J. Mossino, F. Murat, A. Sili, On the Junction of Elastic Plates and Beams, C. R. Acad. Sci. Paris Sér. I Math. 335 (2002), n.8, 717-722.

[9] A. Gaudiello, R. Monneau, J. Mossino, F. Murat, A. Sili, Junction of Elastic Plates and Beams, ESAIM Control Optim. Calc. Var., to appear.

[10] A. Gaudiello, E. Zappale, Junction in a Thin Multidomain for a Fourth Order Problem. $M^{3} A S$ : Math. Models Methods Appl. Sci., to appear.

[11] I. Gruais, Modélisation de la jonction entre une plaque et une poutre en élasticité linéarisée, RAIRO Modl. Math. Anal. Numr. 27 (1993), n.1, 77-105.

[12] V.A. Kozlov, V.G. MA'zya, A.B. Movchan, Asymptotic analysis of Fields in a Multi-Structure, Oxford Mathematical Monographs. Oxford Science Publications. The Clarendon Press, Oxford University Press, New York (1999).

[13] H. LE Dret, Problèmes variationnels dans les multi-domaines: modélisation des jonctions et applications. Research in Applied Mathematics, 19. Masson, Paris, (1991).

[14] G. Panasenko, Asymptotic Analysis of Rod Structures. Kluwer Academic Publishers, (2003). 
[15] P.A. Raviart, J.M. Thomas, Introduction à l'analyse numérique des équations aux dérivées partielles. Dunod, Paris, (1998).

[16] M. Vanninathan, Homogenization of Eigenvalue Problems in Perforated Domains, Indian Acad. Sci. (Math. Sci.) 90 (1981), 3, 239-271.

[17] L. Tartar, Cours Peccot, Collège de France (March 1977). Partially written in F. Murat, H-Convergence, Séminaire d'analyse fonctionnelle et numérique de l'Université d'Alger (1977-78). English translation in Mathematical Modelling of Composite Materials, A. Cherkaev and R.V. Kohn ed., Progress in Nonlinear Differential Equations and their Applications, Birkhäuser - Verlag (1997), 21-44. 